
22 intensities. Additionally, the compressive strains and transmitted impulses increase

\section{Compressive Response of Sandwich Plates to Water-based Impulsive Loading}

Siddharth Avachat and Min Zhou ${ }^{1}$

The George W. Woodruff School of Mechanical Engineering, School of Materials Science and Engineering, Georgia Institute of Technology, Atlanta, GA 30332-0405, USA

\section{Abstract}

The compressive response of sandwich plates with Polyvinyl Chloride (PVC) foam cores and aluminum facesheets to water-based impulsive loading is analyzed using an instrumented impulsive loading apparatus called the Underwater Shock Loading Simulator (USLS) and a fully-dynamic 3-D computational framework. The loading conditions analyzed are similar to those in underwater blasts. The study focuses on the overall deformation, strain recovery and impulse transmission which are quantified as functions of structural attributes such as core density, front and backface masses and incident impulsive load intensity. Measurements obtained using high-speed digital imaging and pressure and force sensors allow the computational models to be calibrated and verified. Quantitative loading-structure-performance maps are developed between the response variables and structural and load attributes. The results reveal that core density has the most pronounced influence on core compressive strain and impulse transmission. Specifically, for severe impulse intensities, a $100 \%$ increase in core density leads to a $200 \%$ decrease in compressive strain and a 500\% increase in normalized transmitted impulse. On the other hand, structures with low density cores are susceptible to collapse at high impulse

\footnotetext{
${ }^{1}$ To whom correspondence should be addressed, Tel: 404-894-3294, Fax: 404-894-0186, Email: min.zhou@gatech.edu
} 
23 monotonically as the mass of the frontface increases, but are unaffected by backface mass. For

24 the same core density, a $100 \%$ increase in facesheet thickness leads to a $25 \%$ and $50 \%$ increase

25 in the core strain and normalized transmitted impulse, respectively. The results and performance

26 maps are useful for designing marine structures with restricts, such as hull sections and pipelines

27 backed by water or machinery.

\section{1. Introduction}

Marine vessels operate in severe environments with temperature extremes, transient loads

30 and corrosive sea water. In addition to operational loads, the structures are required to withstand

31 accidental hydrodynamic impulsive loads due to surface and sub-surface blasts and weapons

32 impact. Sandwich composites can provide good blast mitigation due to their high strength-to-

33 weight ratios and high shear and bending resistances. Previous research on the dynamic behavior

34 of sandwich composites has focused on low velocity contact-based loads such as drop weight

35 and projectile impact [1-8]. It is found that the overall deflection experienced by sandwich plates

36 is significantly lower than monolithic plates of equivalent mass. Additionally, the forces and

37 impulses transmitted by sandwich structures are significantly smaller than those transmitted by

38 monolithic structures [9-11]. Recent assessments of blast-loaded marine structures show that

39 fluid structure interaction (FSI) effects play an important role in response and can be exploited to

40 improve the blast mitigation capability [13-22]. The deformation and failure of sandwich

41 structures subjected to underwater impulsive loads is complicated due to competing damage

42 mechanisms, complex failure modes, interfacial effects and material heterogeneity. The

43 facesheets have a dominant effect on the rigidity of the sandwich structure and provide

44 protection from environmental conditions while the core governs the energy absorption by, and

45 impulse transmission, through the structure. In addition, load intensities, boundary conditions, 
46 and operating environments all influence deformation and failure. Despite recent advances in

47 understanding, several key issues remain unresolved.

48 The objective of the present combined experimental and numerical study is to

49 characterize the behavior of structural foams subjected to underwater impulsive loads and

50 delineate the role of core compressibility and facesheet thickness on the response of sandwich

51 plates. The focus is on quantifying the compression and impulse transmission characteristics of

52 PVC foams with a range of densities under loading of water-based high-intensity impulses

53 generated using a recently developed experimental setup called the Underwater Shock Loading

54 Simulator (USLS). The loads mimic the high-pressure, exponentially-decaying impulses that are

55 generated during underwater explosions. As shown in Figure 1, the USLS consists of a 56 projectile-impact-based impulsive loading system, a water chamber, a target holder and a safety

57 enclosure. In-situ measurements of the material response are obtained using high-speed digital

58 imaging and force transducers, providing an opportunity to assess the role of core density and

59 strength on blast resistance during events mimicking an underwater detonation.

\section{2. Instrumented underwater impulsive loading apparatus}

61 Gas gun impact has been successfully used to generate impulsive loading through water

62 [12-16]. To obtain controlled loading and simulate different water-structure contact conditions,

63 the Underwater Shock Loading Simulator (USLS) in Figure 1 is designed to provide a variety of

64 load configurations with quantitative diagnostics [12-14]. Important features of this facility

65 include the ability to generate water-based impulsive loading of a wide range of intensity, the 66 ability to simulate the loading of submerged structures, and integrated high-speed photographic

67 and laser interferometric diagnostics. Figure 2 shows a schematic illustration of the cross-section 68 of the USLS. 


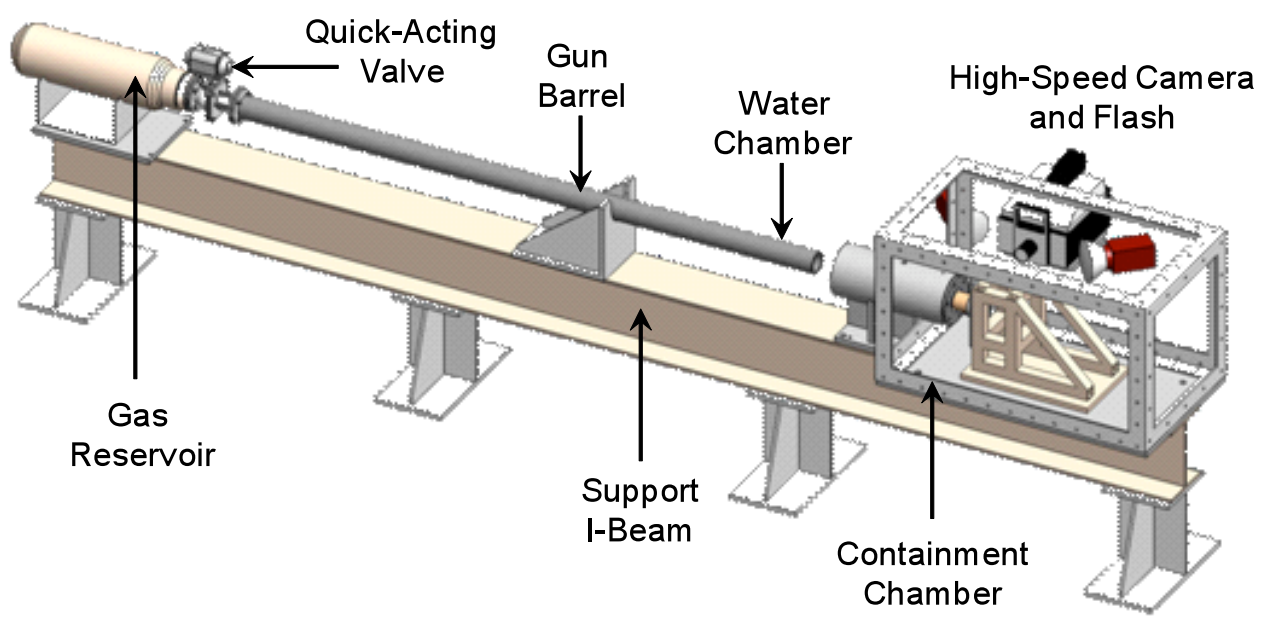

Figure 1 A schematic illustration of the Underwater Shock Loading Simulator (USLS) and a 71 photograph of the facility.

72 The shock tube is an $800 \mathrm{~mm}$ long cylinder which is horizontally mounted and filled with water.

73 It is made of steel and has an inside diameter of $80 \mathrm{~mm}$. A thin piston plate is mounted at the

74 front end and the specimen is located at the rear end. A projectile is accelerated by the gas gun

75 and strikes the piston plate, generating a planar pressure pulse in the shock tube. The impulsive

76 load that impinges on the target induces deformation in the specimen at strain rates up to $10^{4} \mathrm{~s}^{-1}$.

77 Projectile impact velocities in the range of $15-150 \mathrm{~ms}^{-1}$ are used to delineate the effect of loading

78 rate on the deformation and failure behavior of the structures analyzed. This velocity range

79 corresponds to peak pressures between 15 and $200 \mathrm{MPa}$, which are comparable to pressures

80 observed in underwater explosions [17-19]. The metal platens have a thickness of $10 \mathrm{~mm}$ and a

81 diameter of $100 \mathrm{~mm}$; while the foam specimens have a thickness of $50 \mathrm{~mm}$ and a diameter of 70

$82 \mathrm{~mm}$ 


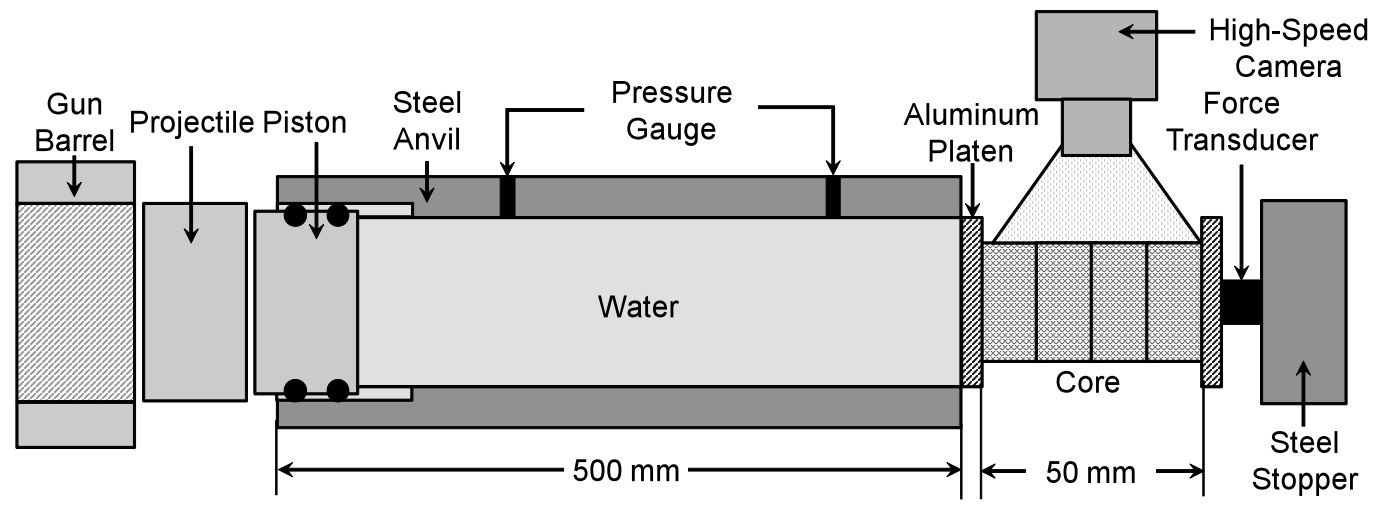

84 Figure 2 A schematic illustration of the dynamic compression "Dynacomp" test setup within the 85 Underwater Shock Loading Simulator (USLS).

86 The uniaxial compressive loading setup developed for this analysis is referred to as the

87 "Dynacomp" setup. Here, an aluminum platen is held in contact with water on one side of the

88 platen and a deformable core on the opposite side of the platen. This deformable core is

89 supported by another aluminum platen which rests on a force transducer embedded in a $25 \mathrm{~mm}$

90 thick steel plate. A flange is designed to ensure that the foam core is always in contact with the

91 aluminum platens on both the impulse side and the opposite side and is held normal to the

92 platens. Care is taken to ensure that there is no slippage between the platens and the core. The

93 compressive strain of the foam core is obtained via high-speed digital imaging and the

94 transmitted impulse is measured using a high dynamic range force transducer. These two

95 parameters provide a description of the compressive response and help quantify the blast

96 mitigation capability of each core configuration. Additionally, the front and backface thicknesses

97 can be varied to evaluate the effect of both variables on the foam core.

98 According to Taylor's analysis of one dimensional blast waves [20] impinging on a light,

99 rigid, free standing plate, the pressure in the fluid at a distance $r$ from an explosive source

100 follows the relation $p(t)=p_{0} \exp \left(-t / t_{0}\right)$, where $p_{0}$ is the peak pressure, $t$ is time and $t_{0}$ is the

101 pulse time on the order of milliseconds. The area under the pressure-time curve is the impulse 
102 carried by the wave and is given by $I_{0}=\int_{0}^{t} p(t) d t=p_{0} t_{0}$. For a free standing plate of areal mass

$103 m$, the impulse transferred to the plate is $I_{T} / I_{0}=\psi^{(\psi / 1-\psi)}$, where $\psi=\rho_{w} c_{w} t_{0} / m$, and $\rho_{w}$ is the

104 density of water and $c_{w}$ is the speed of sound in water. This FSI parameter is an important aspect

105 of Taylor's analysis because it helps to delineate the effects of a pressure pulse applied

106 instantaneously versus the effects of a pressure pulse decaying over a certain time period. It has

107 been shown that this FSI effect can be exploited to improve the blast mitigation capability of

108 structures subjected to transient loads [21,22]. It can be deduced that the impulse transferred to a

109 plate is highly dependent on the mass of that plate. An incoming wave will transfer a very small

110 impulse to a light plate supported by a core with very low strength. Conversely, if the core is

111 strong and resistant to deformation, a larger impulse is transferred to the structure than that

112 predicted by Taylor's analysis. For the upper limit, i.e., an infinitely heavy plate subjected to an

113 intense shock, the entire incident impulse is transferred to the plate. For the loading configuration

114 considered here, the frontface is supported by a core and backface. The backface is fitted with a

115 force transducer and the entire assembly is prevented from moving by a heavy steel plate. Xue

116 and Hutchinson [8] provided a correction to account for a "pushback" effect when the frontface

117 of areal mass $m_{f}$ resists motion by virtue of being supported by a core with compressive yield

118 strength $\sigma_{Y}^{c}$ such that

$$
\bar{I}_{T}=I_{T} / I_{0}=\psi^{(\psi / 1-\psi)}+0.63 \sigma_{Y}^{c} / p_{0}\left(1-\psi^{(\psi / 1-\psi)}\right),
$$

120 and the momentum/area transferred to the core and backface being

$$
\bar{I}_{B}=I_{B} / I_{0}=1.82 \sigma_{Y}^{c} / p_{0}\left(1-\psi^{(\psi / 1-\psi)}\right)
$$


122 The impulse acquired by the frontface of a sandwich structure is $\bar{I}_{F}=I_{F} / I_{0}=I_{T} / I_{0}-I_{B} / I_{0}$. It

123 should be noted that Xue and Hutchinson's work is applicable to relatively weak, perfectly

124 plastic cores that provide a uniform stress-saturated compressive strain response. Additionally,

125 Taylor's $I_{T} / I_{0}$ relation is independent of impulsive load intensity while Xue and Hutchinson's

$126 I_{T} / I_{0}$ relation is dependent on the peak pressure of the incident impulse as well as the yield

127 strength of the core, leading to a loss in generality. In the Dynacomp setup, the backface is

128 essentially immovable and the force transducers fitted to the backface enable the calculation of

129 impulse transmitted through the thickness of the sandwich plate. The reaction force histories can

130 be converted to impulses transmitted via $I_{B}=\int F \cdot d t / A$, where $F$ is the reaction force and $A$ is

131 the area under loading. A normalized transmitted impulse is then expressed as $\bar{I}_{B}=I_{B} / I_{0}$. The

132 transmitted impulse $\bar{I}_{B}$ is an important metric to evaluate the blast mitigation capability of a

133 sandwich structure. It can be inferred from eqn. (2) that a lower value of $I_{B} / I_{0}$ for a specific

134 incident pressure pulse corresponds to better blast mitigation capability of a particular core and

135 the higher the FSI parameter. 

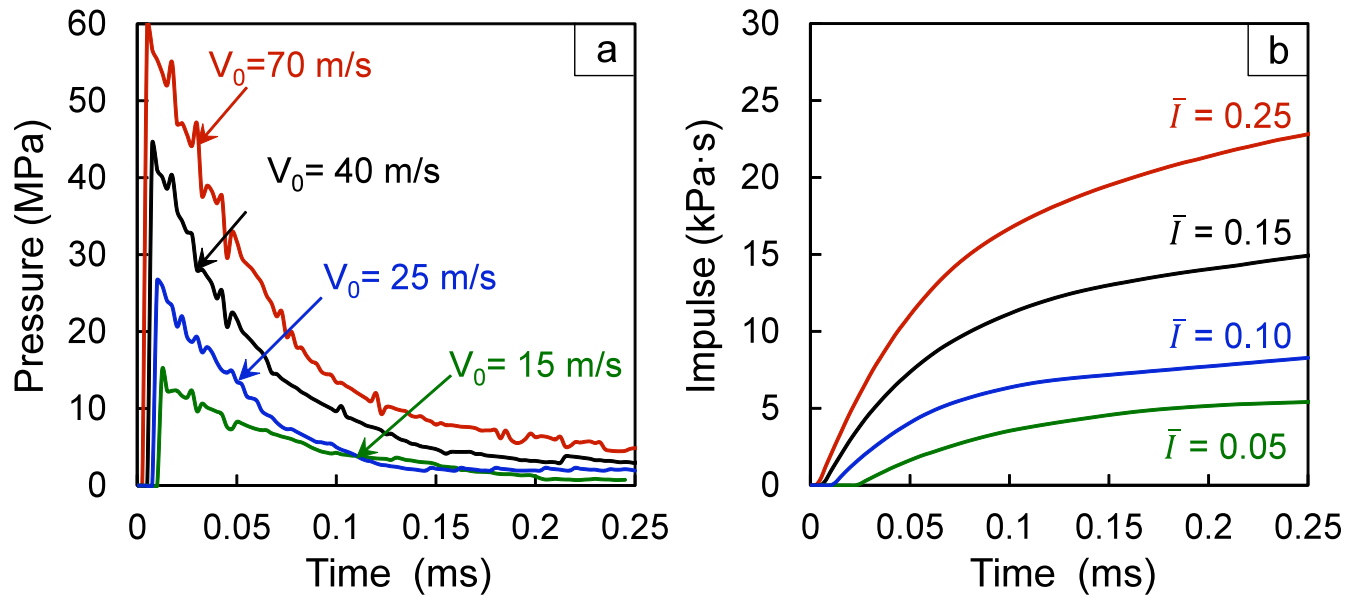

137 Figure 3 (a) The pressure profiles of impulsive waves in the water chamber measured in 138 experiments for four different projectile velocities; (b) the corresponding normalized incident 139 impulses $(\bar{I})$.
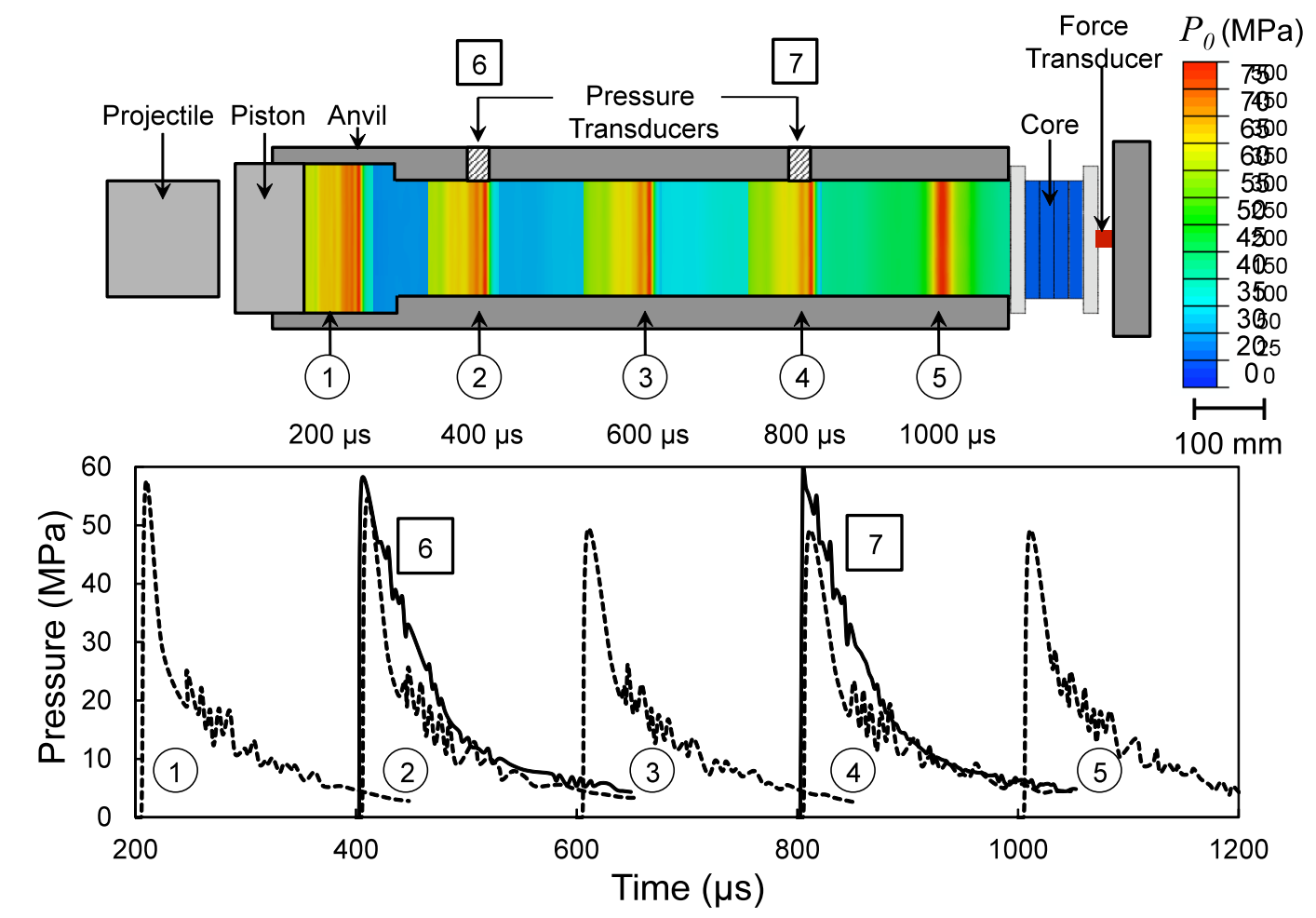

141 Figure 4 Cross-sectional view of the Dynacomp setup showing the distributions of 142 experimentally measured (square-boxed numbers) and numerically calculated (circled numbers) 143 pressure distributions at different locations for an impulsive wave generated with a projectile 144 velocity of $70 \mathrm{~ms}^{-1}(\bar{I}=0.25)$. 
For the current analysis, the non-dimensionalized incident impulse $\bar{I}$ in the form of

$146 \bar{I}=I_{0} / \rho_{w} c_{w} \sqrt{A}$ is used, where $A$ is the area of loading. Pressures ranging from $10 \mathrm{MPa}$ to 100

$147 \mathrm{MPa}$ can be generated using different projectile velocities. Figure 3 shows experimentally

148 measured pressure histories and corresponding impulses for four different projectile velocities. A

149 comparison of experimentally measured and numerically calculated pressure pulses

150 corresponding to a projectile velocity of $70 \mathrm{~ms}^{-1}$ is provided in Figure 4 . The rise time of the

151 pressure pulses is on the order of $25 \mu \mathrm{s}$ and the decay time is on the order of $250 \mu \mathrm{s}$. The

152 impulsive loads have peak pressures of 18, 28, 43, $59 \mathrm{MPa}$ which approximately correspond to

$153100 \mathrm{~kg}$ of TNT detonating at distances of $12,8,5.5$ and 4.2 meters, respectively based on the

154 relationship proposed by Swisdak [20, 23, 24].

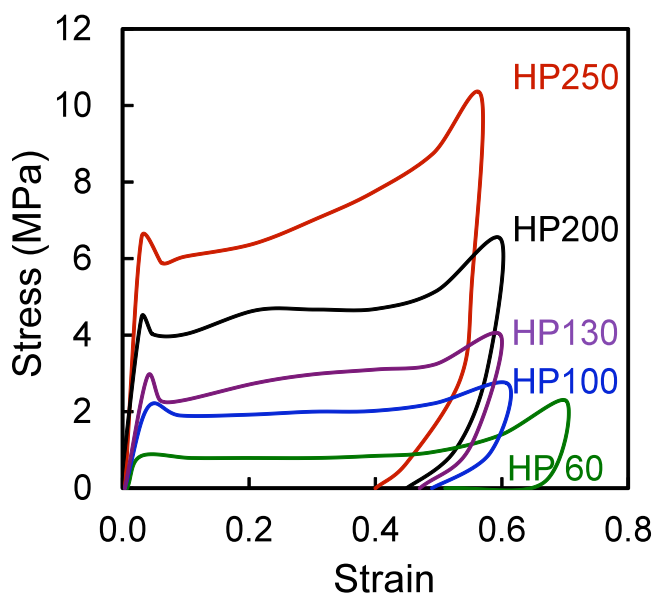

155

Figure 5 Stress-strain curves for the DIAB Divinycell HP foam cores studied [25].

\section{Underwater impulsive loading experiments}

The materials analyzed are structural Poly-Vinyl Chloride (PVC) foams manufactured by DIAB Inc. [26] under the trade name Divinycell HP. These foams are used because their high residual strengths and dimensional stability make them ideal for vacuum bagging and vacuum assisted resin-transfer molding (VARTM). The high strength-to-weight ratio of sandwich structures manufactured using these foams lead to higher vehicle speeds, greater payload 
163 capacities, and reduced power demand, all of which result in better operating economy.

164 Additionally, these structural foams possess high chemical resistance, low water absorbency and

165 good thermal insulation and make ideal core materials in sandwich constructions for marine 166 applications. Here, PVC foams with densities of $60,100,130,200$ and $250 \mathrm{~kg} / \mathrm{m}^{3}$ are studied.

167 The height of the specimen $\left(T_{c}\right)$ is $50 \mathrm{~mm}$ and the diameter $(D)$ is $75 \mathrm{~mm}$. The total thickness of 168 the specimen is $T=T_{c}+T_{f}+T_{b}$ where $T_{c}, T_{f}$ and $T_{b}$ are the core, frontface and backface 169 thicknesses, respectively. The compressive stress-strain responses for the core materials, as 170 obtained by George et al. [25], are shown in Figure 5. The stress-strain relations are linear 171 initially and subsequently show yielding and stress saturation before core densification which 172 leads to a rise in stress. This unique characteristic of the compressive deformation makes the 173 foams especially useful for applications requiring compression and energy absorbency. To 174 compare the effects of different core densities, a normalized density in the form of $175 \bar{\rho}=\rho_{\text {core }} / \rho_{\text {face }}$ is used, where $\rho_{\text {core }}$ is the density of the foam and $\rho_{\text {face }}$ is the density of the 176 facesheet material (aluminum).

177 The circular platens are machined from $12 \mathrm{~mm}$ thick 6061 aluminum alloy plates which 178 have a yield strength of $275 \mathrm{MPa}$ and density of $2700 \mathrm{~kg} \mathrm{~m}^{-3}$. This strength is adequate to ensure 179 that the platens undergo negligible plastic deformation under the impulsive loads considered. In 180 the initial set of experiments, the front and backface thicknesses are $T_{f}=T_{b}=12 \mathrm{~mm}$. To 181 evaluate the effect of frontface mass on the overall response, the thicknesses are increased 182 gradually. The normalized front and backface thicknesses are non-dimensionalized as $T_{f} / T_{c}$ and $183 T_{b} / T_{c}$, respectively, where $T_{c}$ is the core thickness. The facesheet thicknesses are increased 184 gradually and the changes are denoted by $\Delta T_{f}$ and $\Delta T_{b}$, respectively. The values for 
$185 \Delta T_{f}$ and $\Delta T_{b}$ are $4,6,8$ and $10 \mathrm{~mm}$, giving face thickness-to-core thickness ratios $\left(\Delta T_{f} / T_{c}\right)$

186 and $\left(\Delta T_{b} / T_{c}\right)$ of $0.08,0.12,0.16$ and 0.2 . The densities of the platens and the facesheets are

187 much higher than those of the foam cores, so the platens can be considered as effectively rigid.

\section{Numerical model}

The numerical calculations are carried out using the commercial finite element code

190 Abaqus/Explicit [27]. The Mie-Grüneisen equation of state is used to simulate the behavior of

191 water, and the modified Drucker Prager constitutive model developed by Deshpande and Fleck

192 [28] coupled with the damage criterion proposed by Hooputra et al. [29] is used to capture the

193 deformation and fracture in Divinycell HP PVC structural foams. The elements used in this

194 analysis are 3-D 8-noded linear brick elements.

195 4.1 Modeling of water-structure interaction

196 The model consists of a Larangian domain for the solids and an Eulerian domain for the

197 water. In the Lagrangian domain, nodes are fixed within the material and nodal displacements

198 track the material deformation. Since each Lagrangian element is always $100 \%$ within a single

199 material, the material boundary coincides with element boundaries. In contrast, Eulerian the

200 domain consist of nodes that are fixed in space and the material flows through the elements that

201 do not experience deformation. Eulerian elements may also be partially or completely void,

202 allowing material to flow into empty space, capturing a crucial aspect of fluid flow. Materials

203 tracked by Eulerian elements can interact with Lagrangian elements through Eulerian-

204 Lagrangian contact algorithms to allow fully coupled multi-physics simulations like fluid-

205 structure interactions. Here, a coupled Eulerian-Lagrangian (CEL) framework is employed, as it

206 allows the severe deformation in water and the FSI to be captured. In addition to simulating the 
207 blast wave propagation in the USLS, the Eulerian formulation also captures the exponentially 208 decaying pressure waves and resulting cavitation at the fluid-structure interface.

209 The response of water is described by the Mie-Grüneisen equation of state

$$
p=\frac{\rho_{0} c_{0}^{2} \eta}{(1-s \eta)^{2}}\left(1-\frac{\Gamma_{0} \eta}{2}\right)+\Gamma_{0} \rho_{0} E_{m}
$$

211 where $p$ is pressure, $c_{0}$ is the speed of sound, $\rho_{0}$ is initial density, $E_{m}$ is internal energy per

212 unit mass, $\Gamma_{0}$ is Grüneisen's Gamma at a reference state, $s=d U_{s} / d U_{p}$ is the Hugoniot slope

213 coefficient, $U_{s}$ is the shock wave velocity, and $U_{p}$ is particle velocity which is related to $U_{s}$

214 through a linear Hugoniot relation: $U_{s}=c_{0}+s U_{p}$. The space enclosed by the anvil shown in

215 Figure 2 is prescribed the properties of water while the space that is outside the anvil is kept as a

216 "void", allowing water to flow into it as a result of high-pressure wave impinging on the target.

217 This has the effect of instantaneously relieving the pressure in the water-chamber in a manner 218 consistent with experimental observations.

\subsection{Constitutive and damage models for PVC foams}

220 Constitutive models for foams often rely on homogenized continuum descriptions of the 221 cellular materials [30, 31]. The PVC foam core used in the experiments is DIAB Divinycell HP 222 [26] with densities of $60,100,130,200$ and $250 \mathrm{~kg} / \mathrm{m}^{3}$. The Deshpande and Fleck crushable 223 foam plasticity model [28] is used to describe the constitutive behavior of the PVC foams. The 224 yield surface for volumetric hardening is defined as

$$
F=\sqrt{q^{2}+\alpha^{2}\left(p-p_{0}\right)^{2}}-B=0,
$$

226 where $p$ is the pressure, $q$ is the von Mises stress, $\alpha=B / A$ is the shape factor of the yield 227 ellipse that defines the relative magnitude of the axes.. The shape factor is specified by 


$$
\alpha=3 k / \sqrt{\left(3 k_{t}+k\right)(3-k)} \text {, where } k=\sigma_{c}^{0} / p_{c}^{0} \text { and } k_{t}=p_{t} / p_{c}^{0} \text {, }
$$

229 where $\sigma_{c}^{0}$ is the initial yield stress in uniaxial compression, $p_{c}^{0}$ is the initial yield stress in

230 hydrostatic compression and $p_{t}$ is the yield strength in hydrostatic tension. Material parameters

231 for the PVC foams are provided by the manufacturer [26]. Experiments performed show that

232 fracture and fragmentation are significant damage mechanisms in composite sandwich structures

233 subjected to underwater impulsive loads. A phenomenological damage criterion proposed by

234 Hooputra et al. [29] is implemented to predict the onset of rupture due to strain localization and

235 to capture the subsequent fragmentation of the core material. The damage model assumes that

236 the equivalent plastic strain at the onset of damage $\left(\bar{\varepsilon}_{D}^{p l}\right)$ is a function of stress triaxiality and

237 equivalent plastic strain rate, i.e. $\bar{\varepsilon}_{D}^{p l}=\bar{\varepsilon}_{D}^{p l}\left(\eta, \dot{\bar{\varepsilon}}^{p l}\right)$, where $\eta=-p / q$ is the stress triaxiality, $-p$

238 is the hydrostatic stress, $q$ is the von Mises equivalent stress and $\dot{\bar{\varepsilon}}^{p l}$ is the equivalent plastic

239 strain rate. The criterion for damage initiation is met when $\omega_{D}=\int d \bar{\varepsilon}^{p l} / \bar{\varepsilon}_{D}^{p l}\left(\gamma, \dot{\bar{\varepsilon}}^{p l}\right)=1$, where

$240 \omega_{D}$ is a state variable that increases monotonically with plastic deformation. At each increment

241 during the analysis, the incremental increase in $\omega_{D}$ is computed as $\Delta \omega_{D}=\bar{\varepsilon}^{p l} / \bar{\varepsilon}_{D}^{p l}\left(\eta, \dot{\bar{\varepsilon}}^{p l}\right) \geq 0$.

242 The evolution of damage is based on fracture energy per unit area dissipated during the damage

243 process. The data for fracture toughness is obtained from experiments carried out by

244 Poapongsakorn and Carlsson [32].

2454.3 Water-tank, projectile, piston, platens and supports

246 The water-tank and supports are made of stainless steel and the piston, projectile and

247 platens are made of aluminum. A Lagrangian formulation is adopted for these components with 248 linear elastic constitutive behavior. 
$250 \quad$ Failure is predicted when the damage operator in the respective case reaches unity. Once

251 this rupture criterion is satisfied, the strengths of failed elements are set to zero. However, the

252 predictions of damage and structural response based on such failure criteria are inherently mesh-

253 size dependent as shown by Needleman and Tvergaard [33] and Gullerud et al. [34]. When the

254 stress-strain diagram exhibits a negative slope, the strain-softening damage tends to localize in a

255 zone that is governed by element size. Since the damage dissipation per unit volume is finite, the

256 vanishing damage zone causes the structure to fail at zero energy dissipation. In the current

257 computational approach, the mesh size selection must ensure satisfactory strain resolution,

258 realistic energy dissipation and must qualitatively reflect the experimentally observed

259 deformation modes.

260 To counteract this spurious mesh dependence associated with material softening, a

261 characteristic element length $L_{E}$ is introduced in ABAQUS. For 3-D elements, $L_{E}$ is the cube-

262 root of element volume. Following damage initiation, an equivalent displacement $\delta$ is

263 introduced such that $\delta=L_{E} \cdot \varepsilon$ and evolves according to $\dot{\bar{\delta}}=L_{E} \cdot \dot{\bar{\varepsilon}}$ until it reaches a critical

264 value. Although mesh refinement is essential for adequate strain resolution, excessive mesh

265 refinement has the adverse effect of yielding anomalously low energy dissipation. As shown in

266 Figure 6, the numerical solution reaches convergence for a mesh width of $w=500 \mu \mathrm{m}$ for an

267 incident impulse of $\bar{I}=0.25$ for the HP60 core (which experiences the highest compressive

268 strain). Consequently, the mesh width selected for this calculation is $w=500 \mu \mathrm{m}$, which is

269 sufficient for numerical convergence (in both, bulk and cohesive elements) but still provides a

270 reasonable approximation of energy dissipated in the process. 


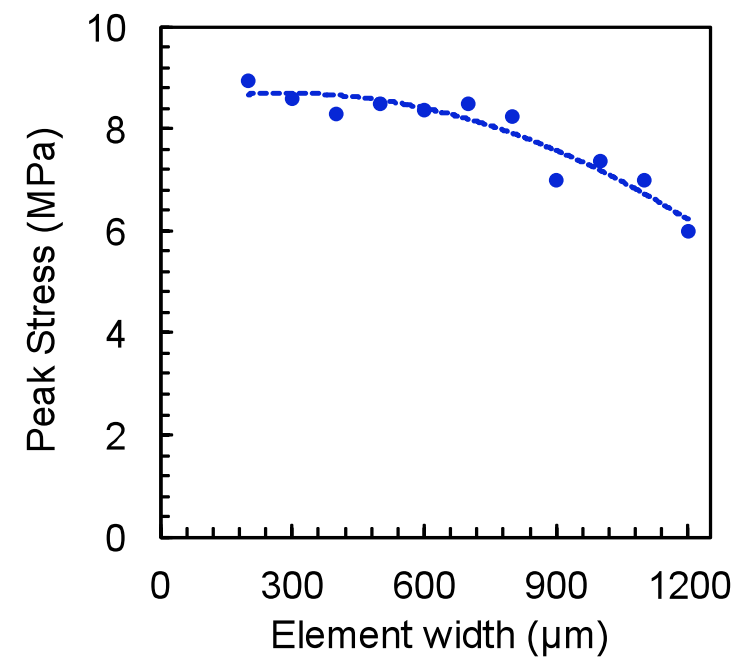

272 Figure 6 Peak stress in the compressed foam as a function of element size for an impulsively 273 loaded HP60 sandwich plate.

\section{5. Loading-structure-performance maps}

275 To fully utilize the potential of sandwich structures, one consideration is to maximize the

276 performance for a give load setting while minimizing the mass. The weight-efficient designs of

277 blast-resistant structures are determined by a number of factors, such as the expected incident 278 load, facesheet and core materials, structural dimensions, geometry and interfacial effects. To 279 quantify the effect of these factors on deformation response, structural indices are developed. 280 Non-dimensional variables are used for quantitative evaluation of the compressive response of 281 the PVC foams and the structural response of composite panels as functions of loading and 282 structural attributes. The performance attribute of interest here is the compressive strain $\varepsilon$, 283 transmitted impulse $I_{B}(\mathrm{kPa} \cdot \mathrm{s})$ and normalized transmitted impulse $\bar{I}_{B}$, the material attribute of 284 interest is the normalized relative density $\bar{\rho}$ and the load is the normalized incident impulse $\bar{I}$. 285 These parameters are varied independently of each other and the performance of each structure is 286 quantified using these parameters. Based on the experiments and numerical simulations reported 
287 here, load-structure-performance maps are developed. These maps can be used to inform

288 structural design with the understanding that they should only be used for the specified material,

289 structural parameter ranges and loading conditions specified.

\section{6. Results and discussion}

2916.1 Experimental results and numerical validation

292 The experimental results are used to calibrate the computational model and evaluate 293 response over a wide range of loading and structural attributes. Figure 7 shows high-speed

294 photographs and corresponding computational contour plots for strain in the HP60 foam 295 subjected to loading with $\bar{I}=0.25$. After the onset of loading, the core undergoes large 296 compressive deformation rather uniformly throughout the thickness. After $750 \mu \mathrm{s}$, strain 297 localization occurs near the frontface and backface. At $1000 \mu \mathrm{s}$, the core compression is 298 complete and strain localization severe throughout the specimen. Figure 8 shows the 299 compressive response of the HP100 foam subjected to loading with $\bar{I}=0.25$. In a manner quite 300 similar to the case for the HP60 foam, the strain in the HP100 core is distributed relatively 301 uniformly throughout the thickness and strain localization occurs at multiple locations. The 302 overall compressive strain is lower than that in the HP60 foam and sites of localized straining 303 coalesce into bands. For both the HP60 and HP100 core materials, the bands are not limited to 304 either the frontface or the backface. Rather, they emanate from the frontface and propagate 305 gradually through the thickness, spanning the whole cross-section.

Figure 9 and Figure 10 show high-speed photographs and corresponding calculated strain

307 fields in HP200 and HP250 foam subjected to loading with $\bar{I}=0.25$, respectively. In both 308 specimens, the strains and resulting strain localization are concentrated at the base of the 309 specimen near the distal face, precipitating inefficient impulse absorption and leading to large 
310 impulse transfer through the foam. The experiments and simulations show reasonable agreement

311 in terms of the rate and extent of compression. Additionally, the simulations reveal

312 characteristics of strain localization and deformation response that are difficult to obtain from

313 experiments, thereby adding valuable insight into the response of each sandwich core. However,

314 larger permanent compressions of the foam cores are observed in the experiments than in the

315 calculations.
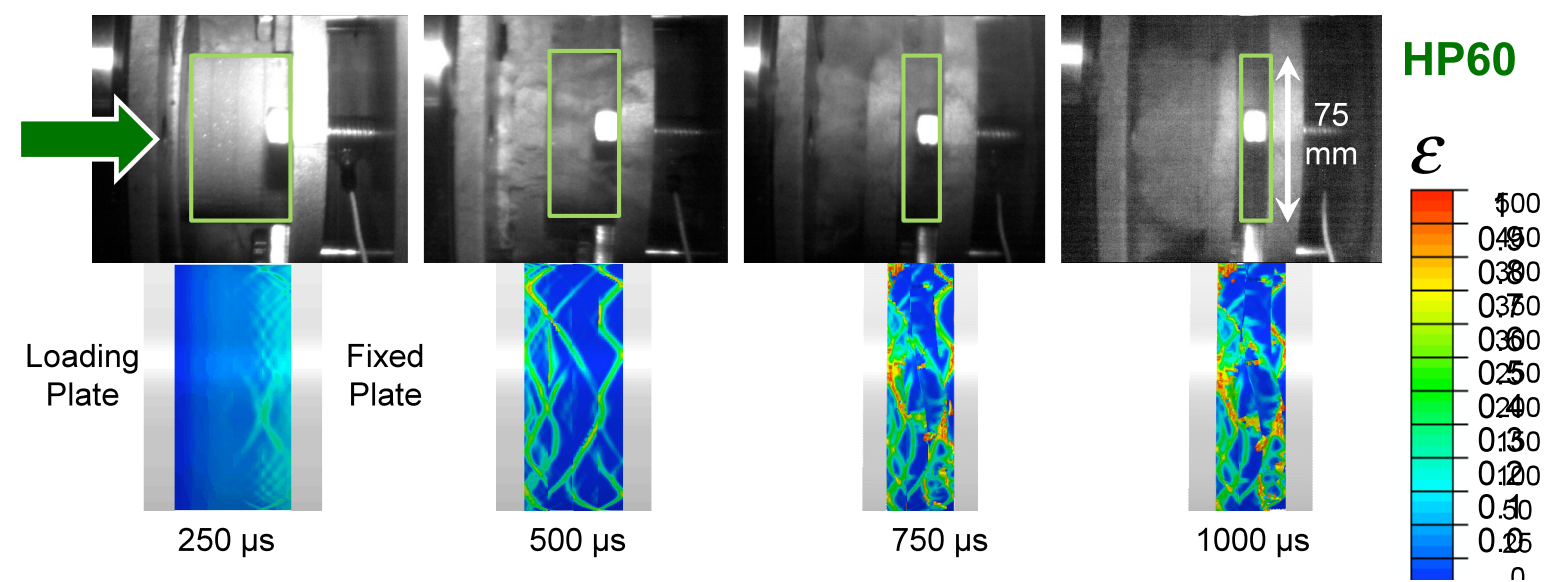

317 Figure 7 A comparison of experimentally measured and numerically calculated strain fields at 318 different times for a sandwich structure with the HP60 core subjected to $\bar{I}=0.25$.
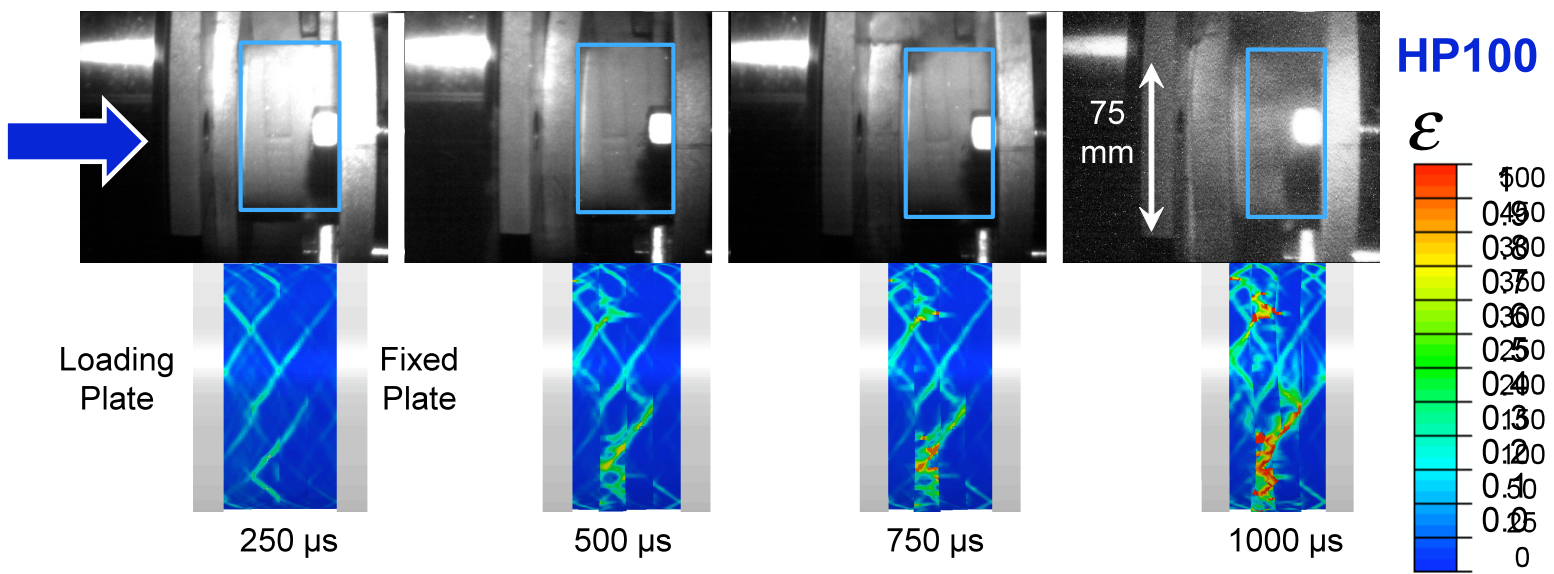

Figure 8 A comparison of experimentally measured and numerically calculated strain fields at 321

different times for a sandwich structure with the HP100 core subjected to $\bar{I}=0.25$. 

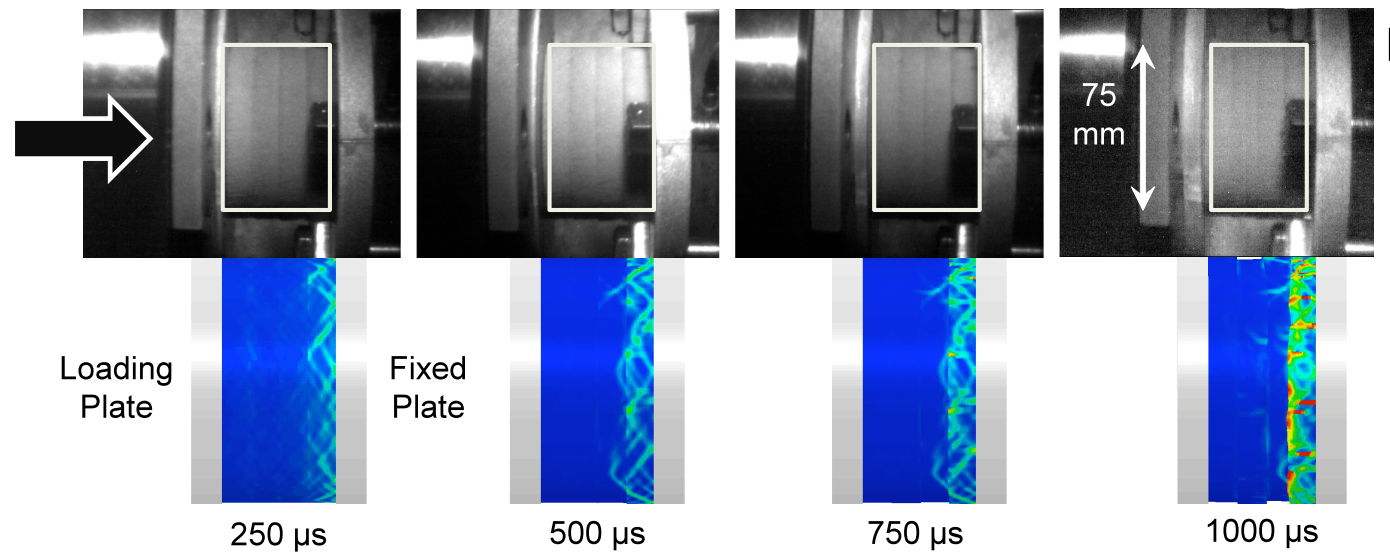

HP200

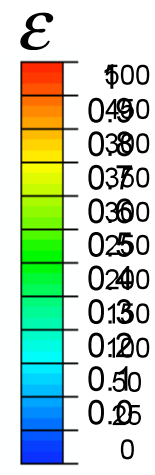

323 Figure 9 A comparison of experimentally measured and numerically calculated strain fields at 324 different times for a sandwich structure with the HP200 core subjected to $\bar{I}=0.25$.

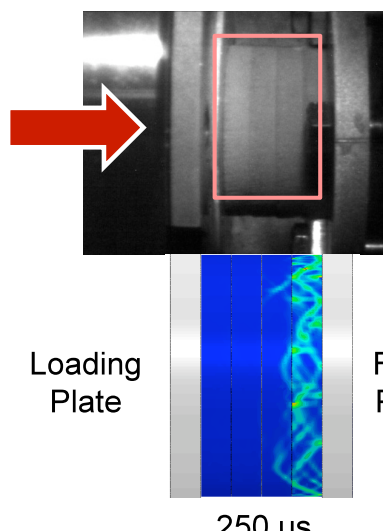

$250 \mu \mathrm{s}$

\subsection{Deformation in the core}

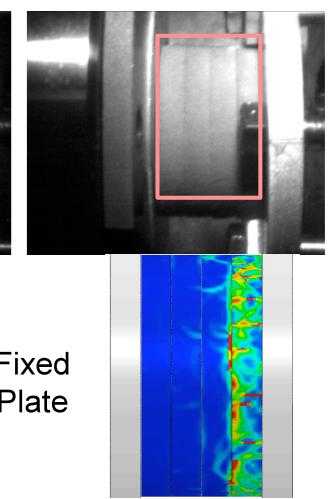

$500 \mu \mathrm{s}$

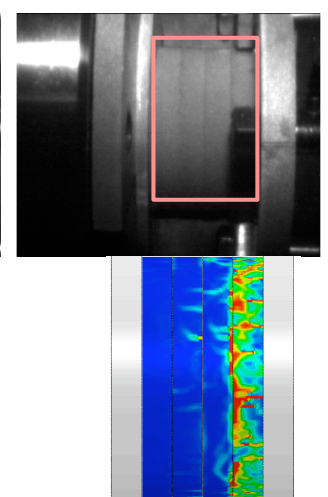

$750 \mu \mathrm{s}$

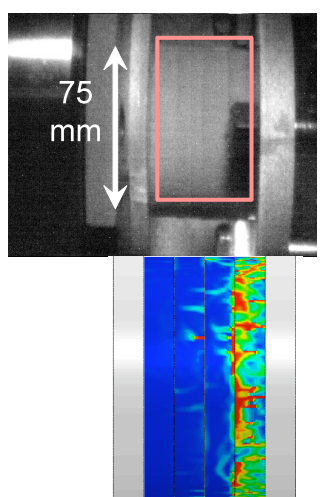

$1000 \mu \mathrm{s}$

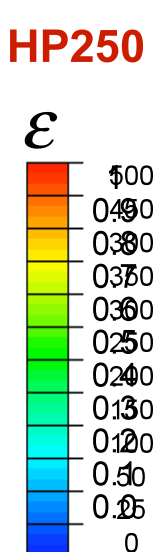

Figure 10 A comparison of experimentally measured and numerically calculated strain fields at different times for a sandwich structure with the HP250 core subjected to $\bar{I}=0.25$.

Figure 11(a-d) shows the time histories of the corresponding compressive strain measured from high-speed digital images for all foams analyzed. For the HP60, HP100 and HP130 foams, rapid compression of the sample occurs immediately after the onset of loading, resulting water leaking from the water tank of the USLS. As the density of the foam increases, both the rate and extent of core compression decrease significantly. Specifically, the HP200 and HP250 cores exhibit negligible compression and essentially behave like monolithic plates,

335 indicating that there is no apparent advantage in using these foam materials in applications in 
336 which energy absorbency or compliance are desired. Instead, these foams may be desirable for

337 applications that require high stiffness.
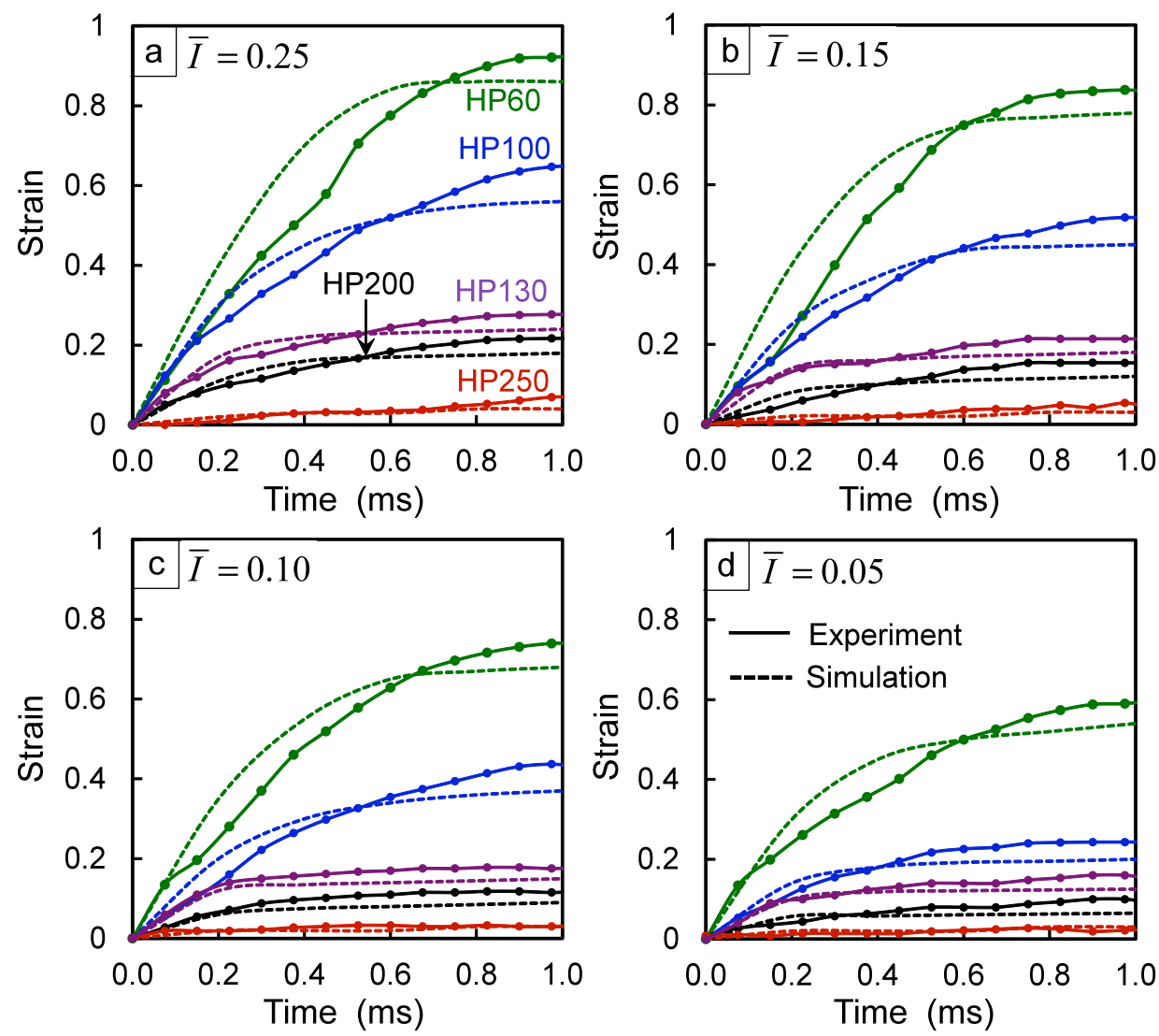

339 Figure 11 Experimentally measured and numerically calculated strain histories for cases with the HP60, HP100, HP130, HP200 and HP250 cores subjected to loading at different intensities.

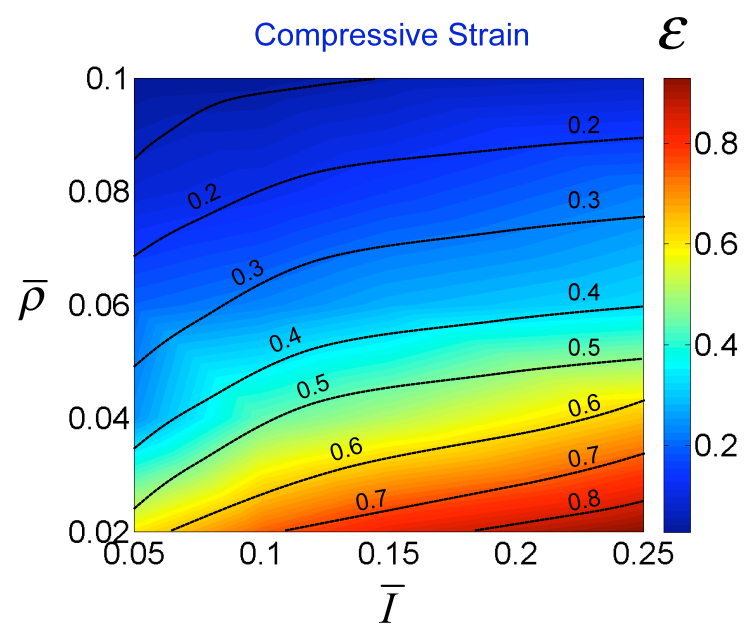

342 Figure 12 Loading-structure-performance map showing compressive strain in the sandwich core as a function of incident impulsive load intensity $\bar{I}$ and normalized density $\bar{\rho}$. 

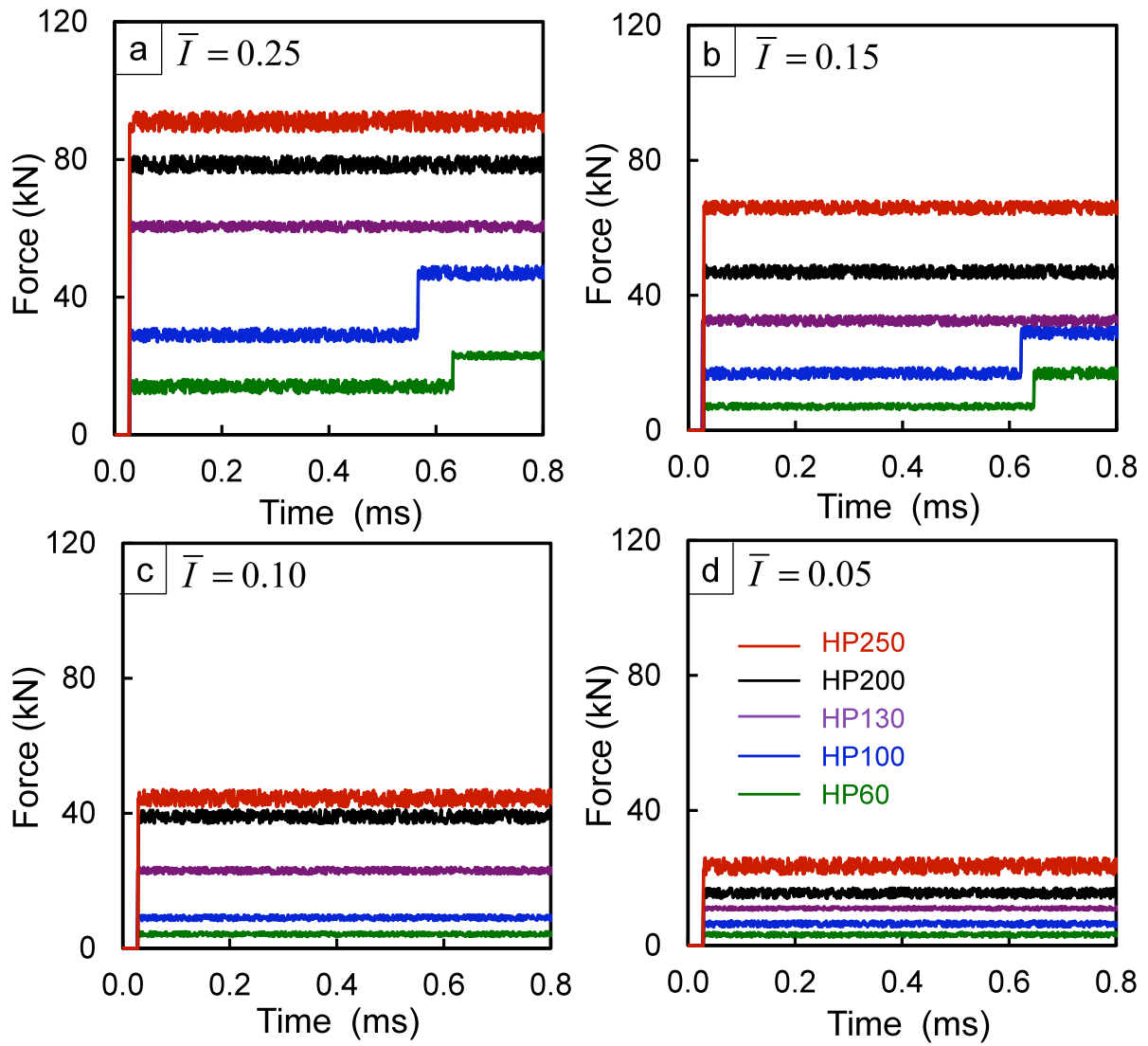

Figure 13 Experimentally measured reaction force histories for sandwich plates subjected to impulsive loading of different intensities.

As the loading intensity increases, both the rate and extent of core compression increase substantially. For the highest load intensity considered, i.e. $\bar{I}=0.25$, the HP60 core experiences maximum compression while the HP100 and HP130 cores show compressions that are $70 \%$ and $40 \%$ of that observed for HP60, respectively. The compressive strains in the HP200 and HP250 cores are $30 \%$ and $10 \%$ of that observed in the HP60 core, respectively. Figure 12 shows the loading-structure-performance map of compressive strain $(\varepsilon)$ as a function of the incident impulse $(\bar{I})$ and relative density $(\bar{\rho})$. At all impulse magnitudes, foams with the lowest relative density experience the highest compressive strain. The compressive strain increases as the relative density increases and as the impulse magnitude increases. The HP250 and HP200 cores exhibit significantly higher resistance to crushing compared with the HP60, HP100 and HP130 
357 foam cores. As the core density increases, the maximum core compression increases 358 monotonically up to $\bar{\rho}=0.05$. With increasing core density, the maximum core compressive 359 strain plateaus at $\bar{I}=0.12$.
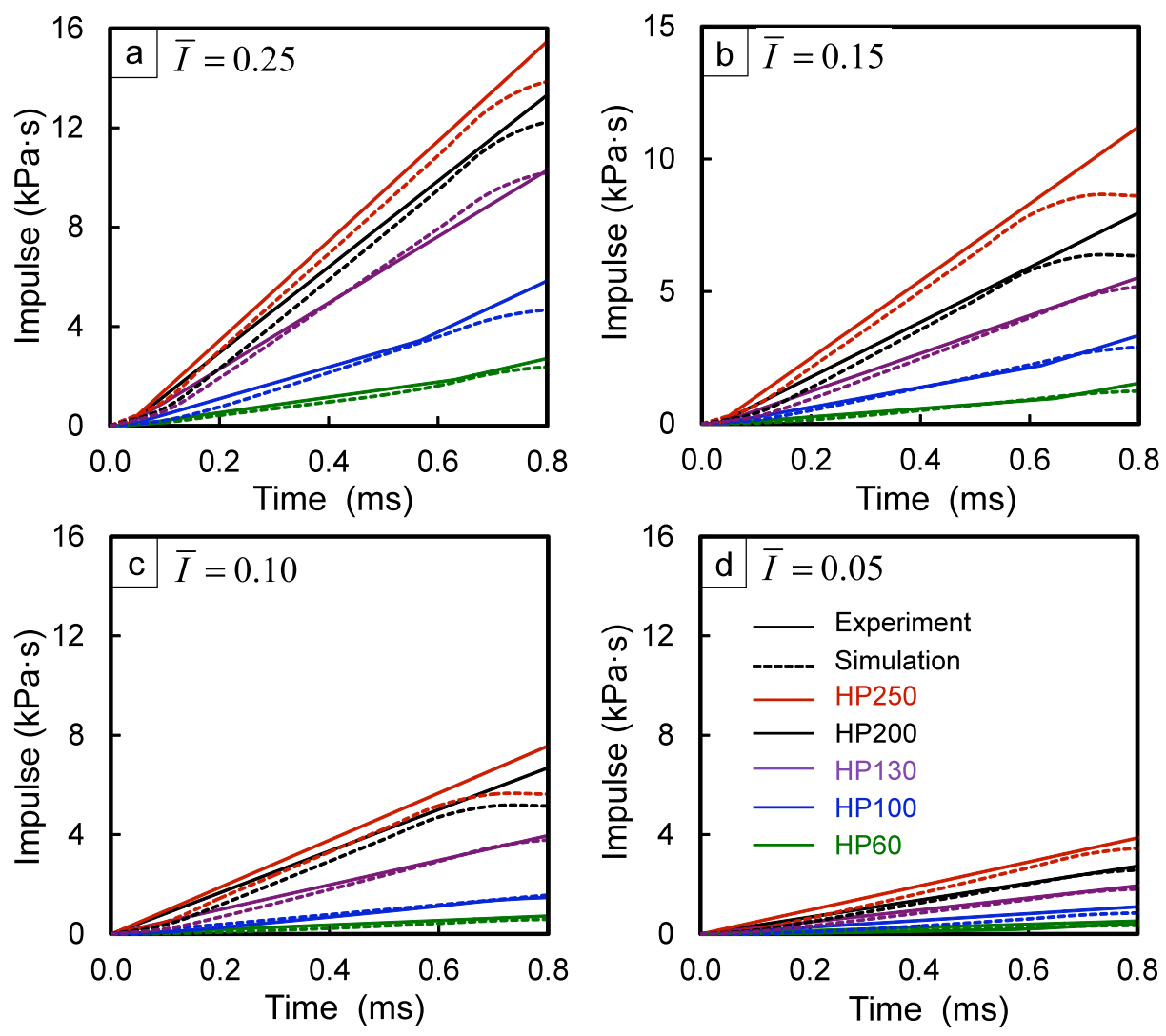

361 Figure 14 Experimentally measured and numerically calculated transmitted impulse histories for 362 Divinycell HP cores subjected to impulsive loading of different intensities.

\subsection{Impulse transmission through the core}

The composite structure that transmits the least impulse at the lowest rate is most

365 desirable. Figure 13(a-d) shows the reaction forces measured by the force transducer for all cores

366 and the input impulse magnitudes. At the higher load intensities $(\bar{I}=0.15$ and 0.25$)$, the

367 crushing and collapse of the core material result in high intensities of transmitted impulse. Core

368 indentation is particularly harmful for sandwich structures as it causes instabilities in the

369 frontface which lead buckling and shear failure as well as increased impulse transmission. Figure 
370 14(a-d) shows the corresponding transmitted impulse histories for all cores and input impulse

371 magnitudes. Lower transmitted impulse indicates better blast mitigation capability. The results

372 show that core density and load intensity both strongly affect impulse transmission. Structures

373 with low density cores consistently outperform structures with high density cores. The effects of

374 core characteristics can be compounded by loading rate.

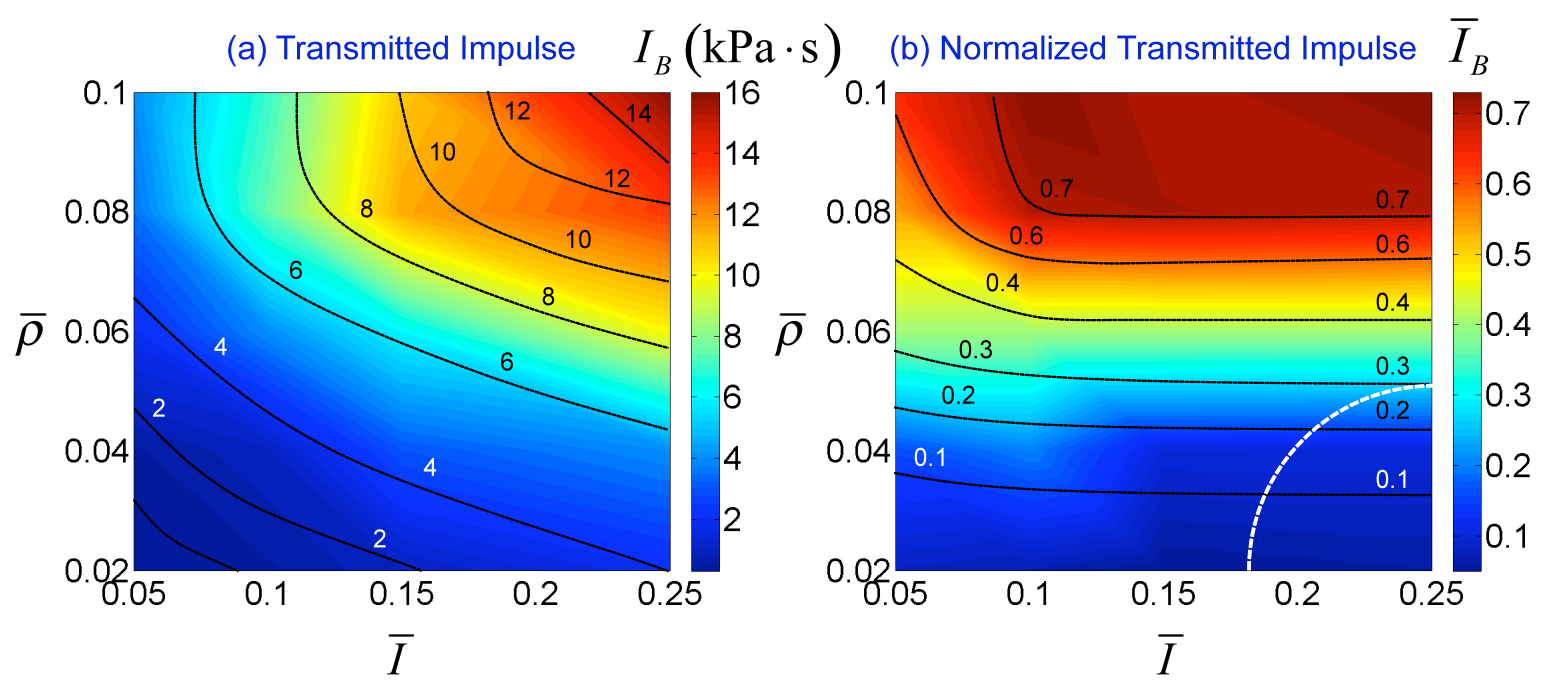

376 Figure 15 Loading-structure-performance map showing (a) transmitted impulse $I_{B}(\mathrm{kPa} \cdot \mathrm{s})$ and

377 (b) normalized transmitted impulse $\bar{I}_{B}$ as functions of incident impulsive load $\bar{I}$ and normalized 378 density $\bar{\rho}$. The region encircled by the white dotted line denotes cores that collapsed under 379 impulses exceeding $\bar{I}=0.10$ as shown in Figure 13.

380 A comparison of Figure 11 and Figure 14 reveals that the rate and extent of core 381 compression correlate with the transmitted impulse, with low density cores undergoing high 382 compressive strains and providing higher blast mitigation than high density cores. These trends 383 are observed at all loading intensities with the low density cores (HP60, HP100 and HP130) 384 transmitting significantly lower impulses than the high density cores (HP200 and HP250). This 385 trend is consistent with deformation fields implied in the contour plots for strain shown earlier. 386 Overall, the uniform distribution of strain and high strain levels in low density cores yield low 387 impulse transmission; and non-uniform distribution of strain and lower strain levels in high 
388 density cores lead to high impulse transmission. Although the total momentum imparted to the 389 sandwich plates is lower for low density cores, the kinetic energy acquired by the frontface is 390 higher in such cases. This results in greater core compression which is detrimental to residual 391 bending stiffness and strength. Since core compression and impulse transmission can pose 392 opposing requirements on structural parameters, an optimum design must balance the competing 393 requirements. Such a design may be different for different load conditions and intensities. Figure 15(a) shows the loading-structure-performance map of transmitted impulse $\left(I_{B}\right)$ 395 as a function of incident impulse $(\bar{I})$ and relative density $(\bar{\rho})$. At all impulse magnitudes, foams with the lowest relative density transmit the least impulses. The transmitted impulses are strongly 397 dependent on relative density and increase with increasing relative density as well as impulse 398 magnitudes. HP60, HP100 and HP130 foam cores exhibit significantly higher blast mitigation 399 capacity in comparison to HP200 and HP250 foam cores. Figure 15(b) shows the normalized 400 transmitted impulse $\left(\bar{I}_{B}\right)$ for all 20 foam core specimens as a function of the incident impulse $401(\bar{I})$ and relative density $(\bar{\rho})$. The variation of $\bar{I}_{B}$ with $\bar{I}$ and $\bar{\rho}$ are quite different from those 402 observed for $I_{B}$. The transmitted impulse as a fraction of incident impulse seems to be only 403 weakly influenced by the incident impulse magnitude but very strongly influenced by the core 404 density. This highlights the fact that in structural design of sandwich composites, the selection of 405 material for the sandwich core is of utmost importance.

406 It should be noted that although low density cores transmit the least impulses, they also 407 undergo high compressive strains and thereby render the structure more susceptible to collapse. 408 The experiments and calculations are in good agreement, indicating that the homogenized 409 Deshpande and Fleck constitutive model [28] in combination with the Hooputra damage criterion 
410 [29] provides a reasonably accurate representation of the deformation in the sandwich core. It

411 should be noted that the Deshpande and Fleck constitutive model slightly overestimates the

412 compliance of the foam core, leading to a higher initial rate of core compression and marginally

413 greater transmitted impulses.

4146.4 Effect of face thickness on deformation and impulse transmission

415 The effect of facesheet thickness is analyzed by systematically varying both the front and 416 back facesheet thicknesses and by evaluating the response of each sandwich core. The facesheet

417 thicknesses considered are $4,6,8$ and $10 \mathrm{~mm}$, giving $\left(\Delta T_{f} / T_{c}\right)$ and $\left(\Delta T_{b} / T_{c}\right)$ of $0.08,0.12,0.16$

418 and 0.2 where $\Delta T_{f}$ and $\Delta T_{b}$ are the changes in front and back face thicknesses respectively.

419 Figure 16 shows a comparison of experimentally observed and calculated compressive response 420 of the structure with the HP100 core with $\left(\Delta T_{f} / T_{c}\right)=0.2$.
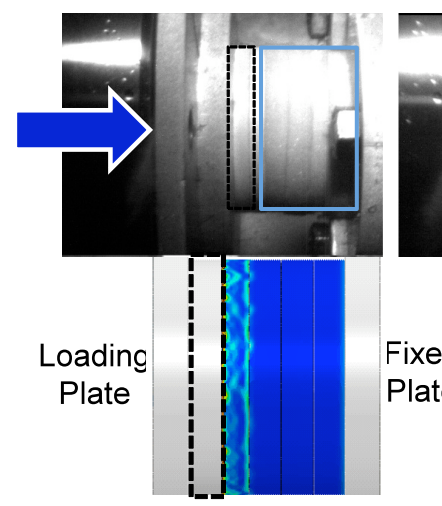

$250 \mu s$
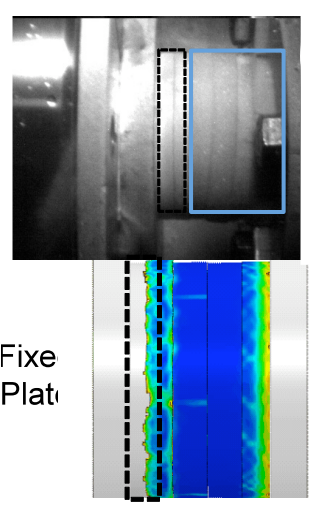

$500 \mu s$

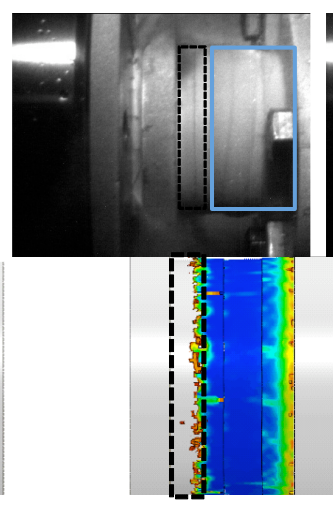

$750 \mu \mathrm{s}$

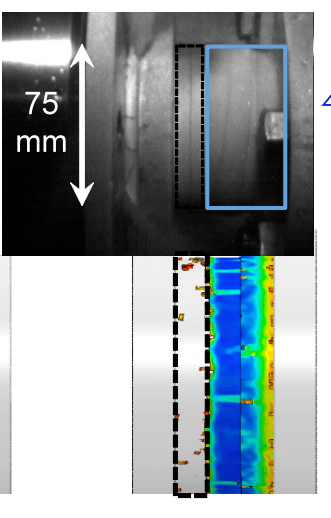

$1000 \mu s$
HP100

$\Delta T_{f} / T_{c}=0.2$

$\mathcal{E}$

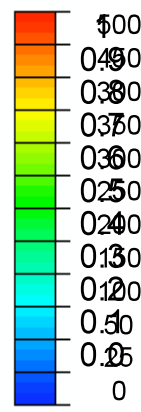

422 Figure 16 A comparison of experimentally measured and numerically calculated strain fields at 423 different times for a sandwich structure with the $\operatorname{HP} 100$ core $(\bar{I}=0.25)$.

424 Strain localizes predominantly near the impulse face and near the distal face due to 425 significant wave reverberations. It is instructive to note the differences between the responses of 426 the HP100 core with $\left(\Delta T_{f} / T_{c}\right)=0.2$ and with $\left(\Delta T_{f} / T_{c}\right)=0$ shown in Figure 8. For 
$427\left(\Delta T_{f} / T_{c}\right)=0$, the compressive strain is relatively uniform throughout the thickness. For $428\left(\Delta T_{f} / T_{c}\right)=0.2$, the compressive strain tends to localize near the facesheets. Figure 17(a) shows

429 the compressive strain and Figure 17(b) shows the history of the corresponding transmitted 430 impulse for structures with the HP100 core and different frontface thicknesses under loading 431 with $\bar{I}=0.25$.
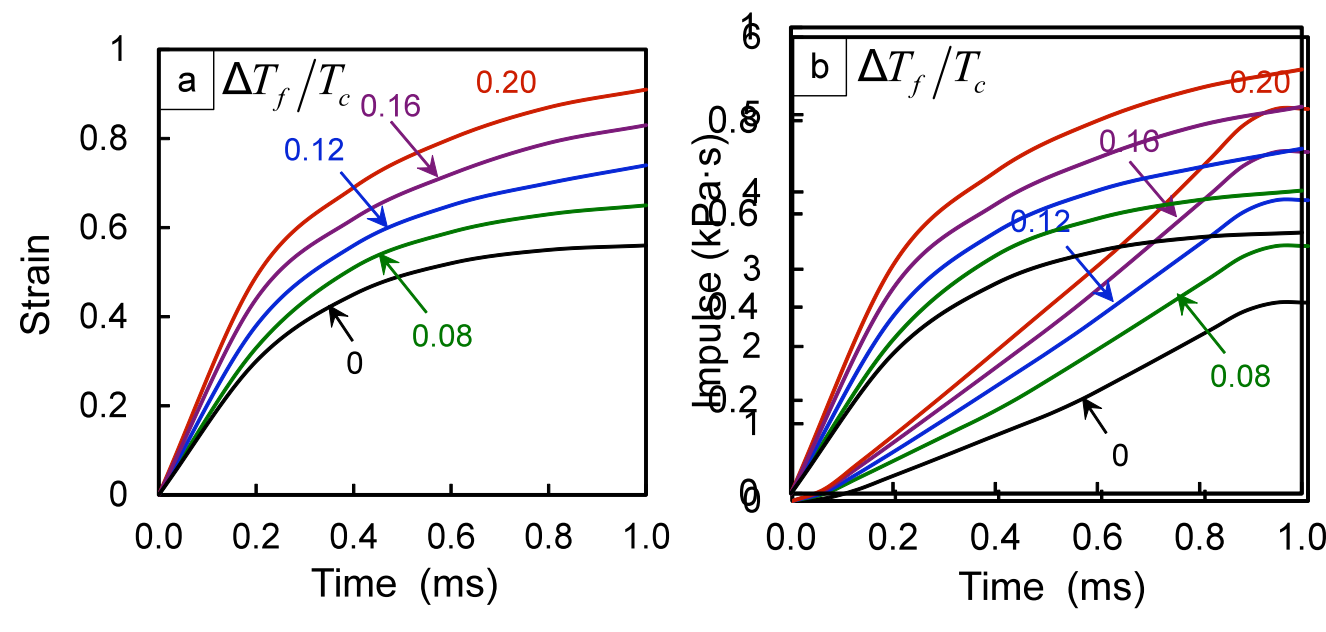

433 Figure 17 Compressive strain and transmitted impulse histories for different $\left(\Delta T_{f} / T_{c}\right)$ values for 434 the HP100 core subjected to $\bar{I}=0.25$.

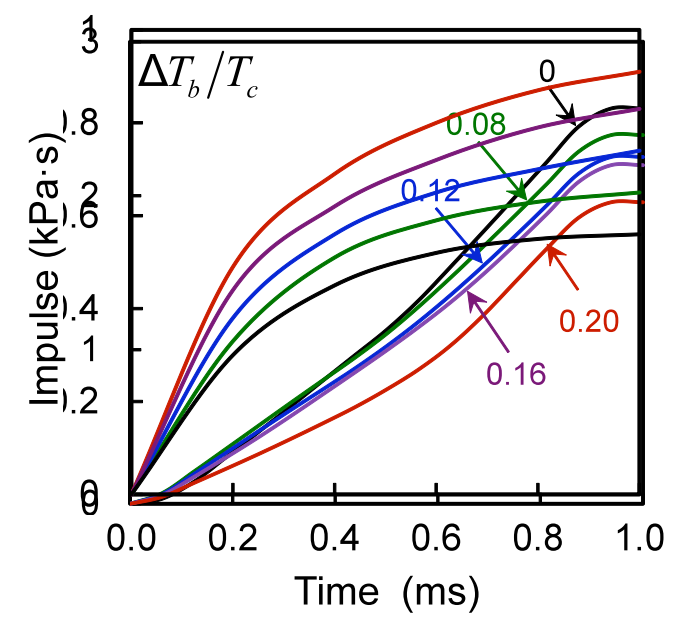

436 Figure 18 Transmitted impulse histories for different ratios between backface thickness and core 437 thickness $\left(\Delta T_{b} / T_{c}\right)$ for the HP100 core subjected to $\bar{I}=0.25$. 
As the frontface thickness increases, the strains increase and the transmitted impulse

439 increases accordingly. Although thicker frontfaces tend to increase impulse transmission,

440 sufficiently strong frontfaces are necessary for structural integrity. Therefore, sandwich

441 structures must balance the strength and mass of the frontface and allowable core compression to

442 control impulse transmission. Figure 18(b) shows the effect of backface thickness on the

443 transmitted impulse. As the backface thickness increases, the transmitted impulse decreases only

444 slightly. Since the benefit is relatively negligible, the influence of backface thickness on

445 structural response is not analyzed further. Figure 19(a) shows the peak compressive strain $(\varepsilon)$

446 and Figure 19(b) shows the normalized transmitted impulse $\left(\bar{I}_{B}\right)$ for all specimens as functions

447 of frontface thickness $\left(\Delta T_{f} / T_{c}\right)$ and relative density $(\bar{\rho})$ for $\bar{I}=0.25$. The results reveal that

448 both core compressive strain and normalized transmitted impulse increases with increasing

449 frontface thickness. For a 10\% increase in frontface mass, the core compressive strain increases

450 by $\sim 5 \%$ and transmitted impulse increases by $\sim 10 \%$. Overall, the core density has a strong

451 influence on blast resistance and the frontface thickness.
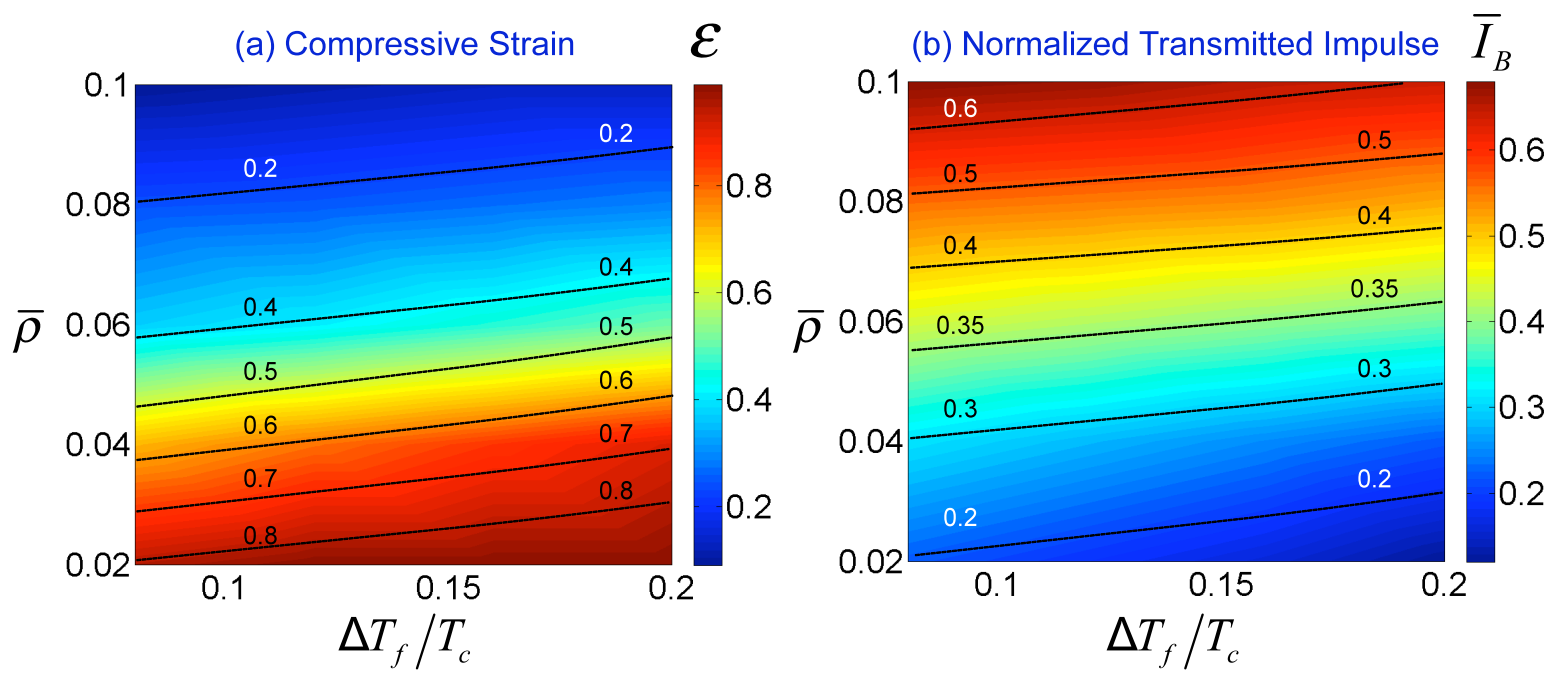
453 Figure 19 Loading-structure-performance map showing compressive strain $\mathcal{E}$ and normalized 454 transmitted impulse $\bar{I}_{B}$ as functions of $\left(\Delta T_{f} / T_{c}\right)$ and normalized density $\bar{\rho}$.

\section{$455 \quad 6.6$ Comparison with analytical model}

A number of analytical models have been developed to extend Taylor's relation for an

457 underwater impulse impinging on a free-standing plate [18]. These relations are generally based

458 on a simplified continuum description of the sandwich core. Figure 20 shows a comparison

459 between the predictions of Xue and Hutchinson's analyical model [8] and the experimental

460 results obtained in the current study. The Xue and Hutchinson approach clearly delineates the

461 benefit of sandwich plates over monolithic plates of equivalent mass and identifies minimum

462 weight designs. This analytical model, based on a continuum description of the core, accurately

463 predicts the transmitted impulses for low core densities, but significantly underestimates the

464 transmitted impulses for high core densities.

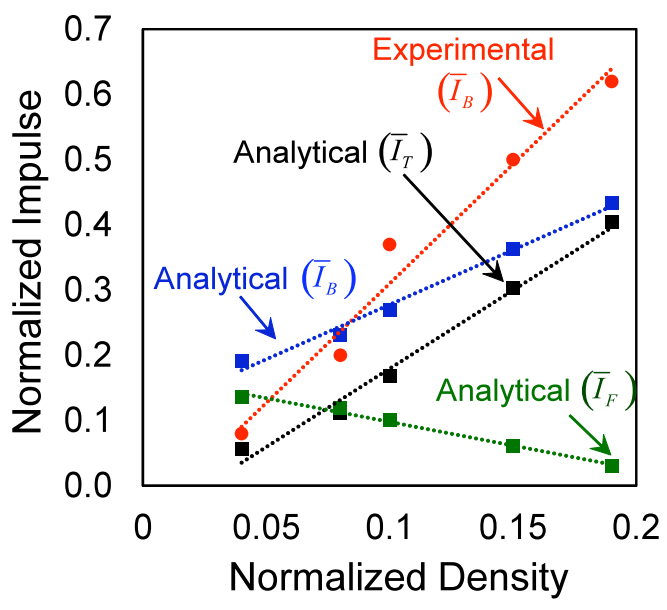

Figure 20 A comparison of normalized transmitted impulse values obtained from experiments 467 and calculated using Xue and Hutchinson's analytical approach [8].

\section{Scaling and structural design}

The underwater blast loading problem involves a wide range of length and time scales 
471 geometry of the structure, and the area under impulsive loading relative to the total area of the

472 structure. The characteristics of high intensity pressure pulses resulting from underwater blasts

473 have been well established through large-scale experiments. In the analysis reported here, the

474 underwater blast loading problem is simplified to delineate the performance of the sandwich core

475 and provide a clear understanding of the role of core thickness, core density and facesheet

476 thickness on blast response. The test specimen is designed such that the structure experiences

477 one-dimensional, uniaxial compressive loading. This eliminates the complexities associated with

478 large scale beam bending and facesheet stretching in blast loaded sandwich plates. The

479 momentum transmitted into the sandwich plate is highly dependent on core strength and density.

480 Denser cores $(\bar{\rho}>0.06)$ better resist the motion of the frontface and lead to greater momentum

481 transfer. At impulse intensities $\bar{I}>0.15$, cores with $\bar{\rho}<0.06$ undergo densification and 482 collapse. 


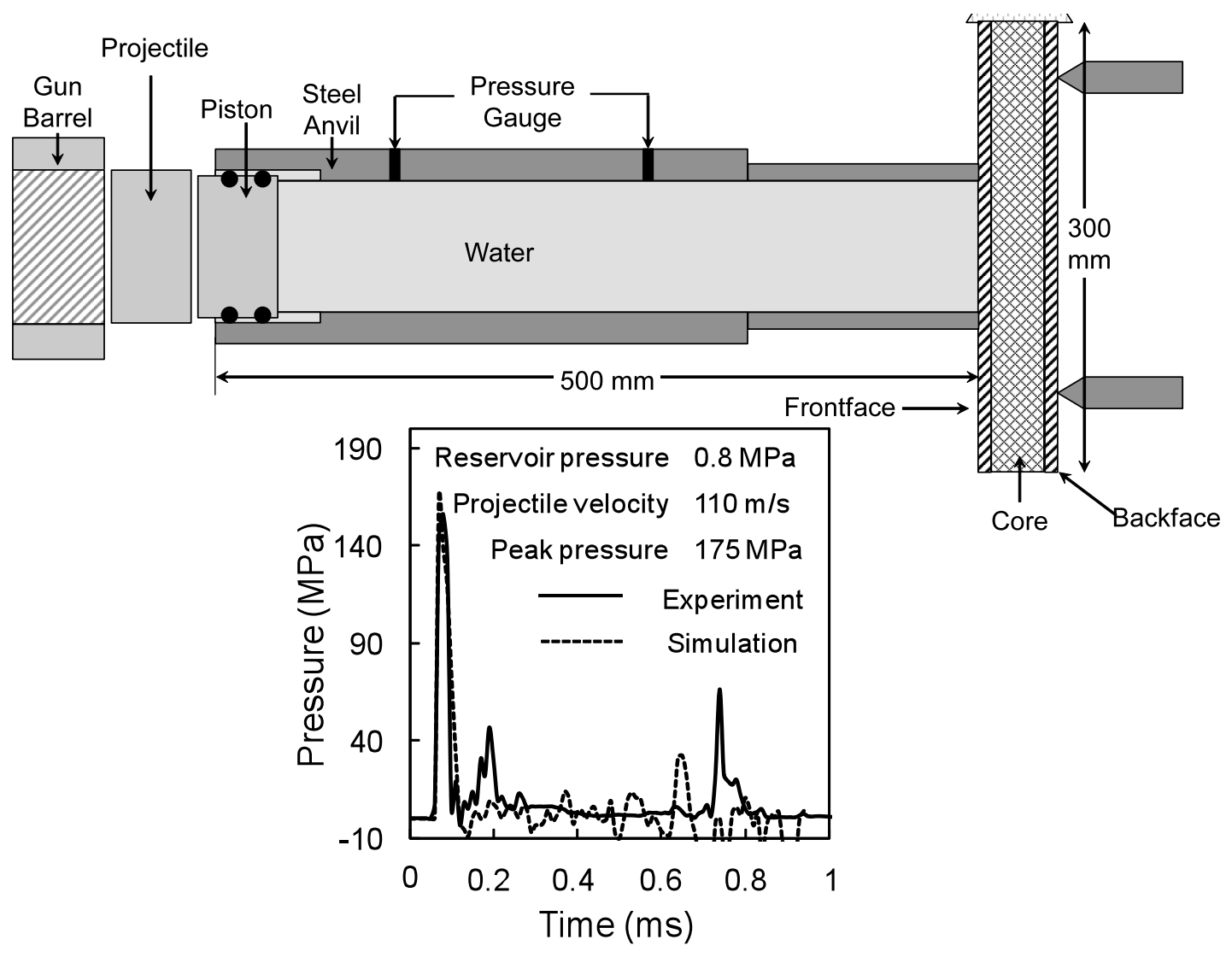

484 Figure 21 A schematic illustration of the simply-supported air-backed loading configuration 485 within Underwater Shock Loading Simulator (USLS).

486 On the other hand, for $\bar{I}>0.15$ and $\bar{\rho}>0.06, \varepsilon$ is 0.2 and almost constant at all loading

487 intensities. The momentum transmitted into the sandwich plates for $\Delta T_{f} / T_{c}<0.1$ is substantially

488 lower than that for $\Delta T_{f} / T_{c}>0.15$. For the same core density, a $100 \%$ increase in facesheet

489 thickness leads to a $25 \%$ and $50 \%$ increase in the core strain and in normalized transmitted

490 impulse, respectively. For a given incident impulse, $\Delta T_{f} / T_{c}>0.15$ result in more severe core

491 compression because the impulse acquired by the frontface increases in proportion to mass. 


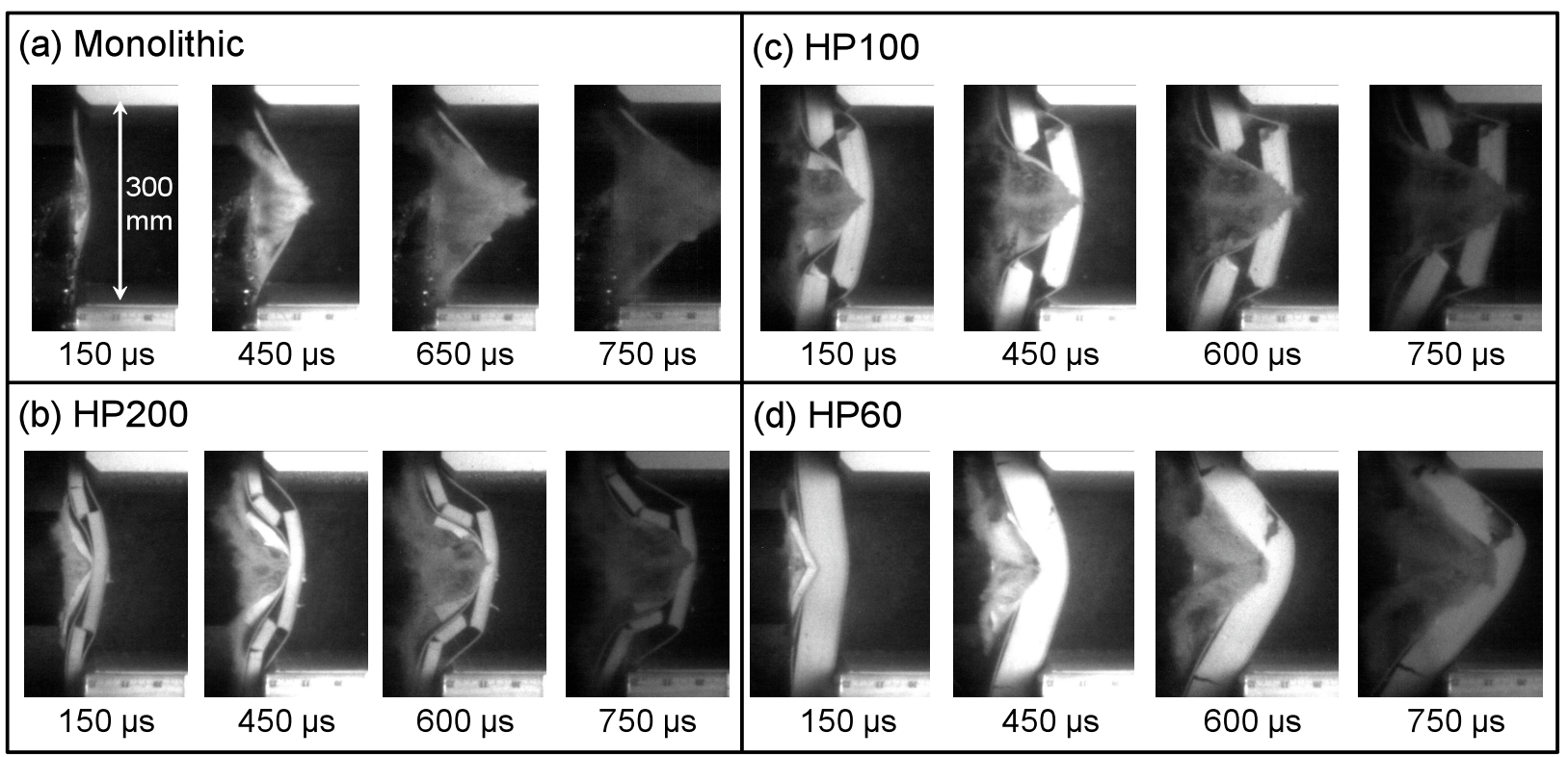

493 Figure 22 Sequence of high-speed photographs showing the deformation in composite structures 494 subjected to $p_{0}=175 \mathrm{MPa}$. The impulse imparted to the frontface causes it to move away at a 495 velocity higher than the allowable dynamic crush rate of the core resulting in large differential 496 displacements which cause frontface fracture and core cracking, but relatively low core 497 compression.

A set of experiments and simulations is carried out to correlate the performance of the

499 sandwich plates under uniaxial compression with the performance under bending of a blast

500 loaded sandwich plate [35]. Figure 21 shows a schematic illustration of the USLS with a simply-

501 supported loading configuration. The sample size considered here is approximately one order of

502 magnitude smaller than composite sections used in ships. The impulsive loads considered in this

503 set of calculations have peak pressures of 40, 90, 140 and $175 \mathrm{MPa}$, which approximately

504 correspond to $100 \mathrm{~kg}$ of TNT detonating at distances of 5.8, 2.83, 1.9 and 1.5 meters,

505 respectively. These impulsive loads are of greater intensity than those analyzed using the

506 Dynacomp setup. The facesheets are made of biaxial E-glass/vinylester composites and the core

507 is PVC foam manufactured by DIAB Inc. [36]. Three PVC foam densities are analyzed: 60, 100

508 and $200 \mathrm{~kg} / \mathrm{m}^{3}$. The designs considered in this analysis have similar areal masses. To compare 
509 the effects of different core densities on response, a relative density $\bar{\rho}$ is defined such that $510 \bar{\rho}=\rho_{\text {core }} / \rho_{\text {face }}$

511 Figure 22 shows a sequence of high-speed photographs of the deformation in different 512 composite structures subjected to $p_{0}=175 \mathrm{MPa}$, which is the highest load intensity considered 513 in this analysis. Figure 22(a) shows the response of monolithic composite plate. The deformation 514 can be divided into two regimes: (1) flexural wave propagation towards the supports and (2) 515 structural deflection. The flexural wave travels towards the supports in a very short time $(\sim 50$ $516 \mu \mathrm{s})$. Figure 22(b) shows the response of a sandwich structure with HP200 core subjected to $517 p_{0}=175 \mathrm{MPa}$. The core fractures in a direction perpendicular to the planar wave and causes 518 considerable core-face debonding in both the front and the back interfaces. Core compression is 519 negligible and fragmentation is observed near the supports. Figure 22(c) shows the behavior of a 520 sandwich structure with HP100 core. The HP100 core fractures at an inclined angle from the 521 loading direction and simultaneously undergoes core compression and crushing. The response of 522 a sandwich structure with an HP60 core is shown in Figure 22(d). Core compression and 523 frontface wrinkling are observed at $t=150 \mu \mathrm{s}$. Core indentation occurs at $t=300 \mu \mathrm{s}$ and the core 524 starts to crack at $t=450 \mu \mathrm{s}$. Damage and deformation in the sandwich structure with a HP60 525 core is significantly lower than those in the other structures. At high load intensities, it appears 526 that the impulse imparted to the frontface causes it to move away at velocities higher than the 527 allowable dynamic crush rate of the core, resulting in large differential displacements which 528 cause frontface fracture and core cracking, but negligible core compression. 

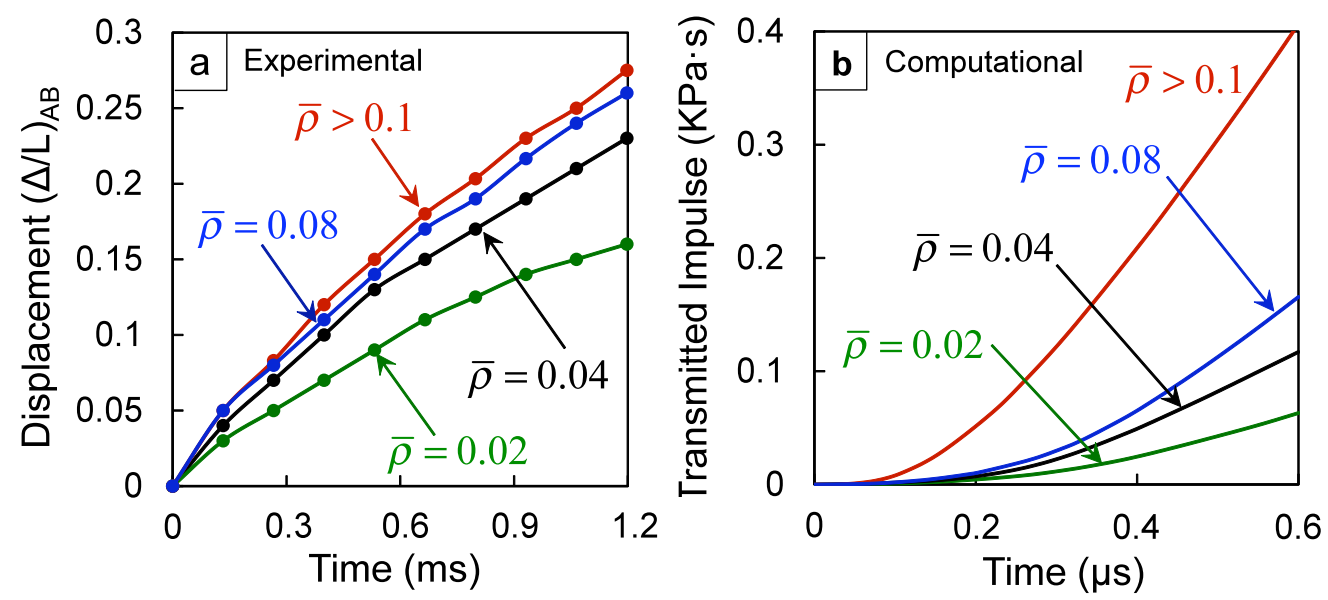

530 Figure 23 (a) Experimentally measured midpoint displacements and (b) computationally 531 calculated measured transmitted impulses as functions of time for air-backed sandwich structures 532 subjected to an impulse with

533 Figure 23(a) shows the midpoint displacements as functions of time for the four different

534 structures. The sandwich structures with HP200 and HP100 cores and the monolithic structures

535 show relatively similar deformation histories reaching a $\Delta / L$ value of 0.25 at approximately the

536 same rate. The sandwich structure with HP60 cores shows superior blast mitigation, deflecting at

537 a lower rate and reaching a $\Delta / L$ value of 0.17 , which is $\sim 60 \%$ of that for the other sandwich

538 structures. The results show that core density and load intensity profoundly affect both the rate

539 and the extent of deformation in the composite structures. The study indicates that structure with

540 low density cores consistently outperform structures with high density cores of equal mass.

541 Lower core density and thicker cores correspond to reduction in velocity due to more significant

542 core compression. Additionally, variations in geometric parameters have an effect on flexural

543 rigidity and deformation. Since a fully 3D, dynamic computational framework is used to in this

544 analysis, structural effects beyond bending, as well as bending, are captured.

545 Minimizing the impulse transmitted to the internal components of marine vessels is of

546 critical importance. The rate of impulse transmission and the magnitude of the transmitted 547 impulse can provide valuable insight into the blast resistance and performance of composite 
structures. Clearly, the composite structure that transmits the least impulse at the lowest rate is most desirable. Figure 23(b) shows the histories of impulses transmitted by air-backed structures subjected to incident impulsive loads of different magnitudes. For an incident impulse with $p_{0}=175 \mathrm{MPa}$, the sandwich structures with HP200, HP100 and HP60 cores transmit $40 \%$,

$55230 \%$ and $20 \%$ of the impulse transmitted by the monolithic composite, respectively. Correlating

553 the rate of impulse transmission with the core characteristics in each case shows that, as the core

554 density decreases and core thickness increases accordingly, the rate of impulse transmission 555 decreases significantly.

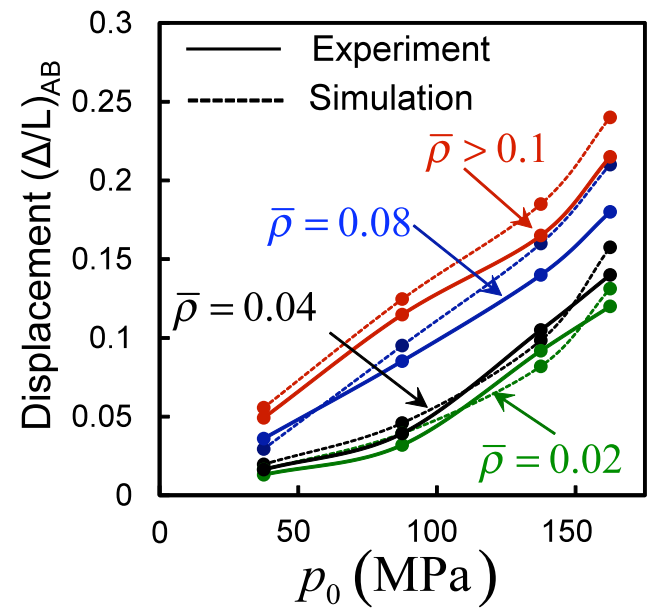

557 Figure 24 Comparison of experimentally measured and numerically calculated mid-plane 558 deflections at $1000 \mu \mathrm{s}$ in air-backed structures as functions of normalized incident impulse $p_{0}$ 559 for different normalized core densities. The results from experiments are in good agreement with 560 those obtained from finite element simulations.

Figure 24 shows the normalized deflection $(\Delta / L)$ as a function of impulse $\bar{I}$ for

562 structures with different normalized relative densities $\bar{\rho}$. A monotonically increasing trend of

563 center deflections with increasing core density is seen and shows reasonable agreement with 564 experiments. At all impulse magnitudes, structures with the lowest relative density experience 565 the least deflections. The deflection increases with increasing relative density and impulse 566 magnitude. The structure with the HP200 core performs only marginally better than monolithic 
567 structures. The HP100 and HP60 cores yield significantly higher blast resistances in comparison

568 to the HP200 core and the monolithic composite. Figure 25(a) shows the loading-structure-

569 performance map of normalized deflection $(\Delta / L)_{A B}$ as a function of impulse $\bar{I}$ and relative

570 density $\bar{\rho}$. As core density increases, the out-of-plane deflection of the sandwich plates increases

571 dramatically. Figure 25(b) shows the loading-structure-performance map of transmitted impulse

572 for air-backed structures $\left(\bar{I}_{B}\right)$ as a function of normalized incident impulse $\bar{I}$ and normalized

573 relative density s $\bar{\rho}$. At all impulse magnitudes, structures with the lowest relative density

574 transmit the least impulse. The transmitted impulse increases with increasing relative density as

575 well as impulse magnitude. HP200 cores perform better than monolithic structures while HP100

576 and HP60 cores exhibit significantly higher blast mitigation in comparison to HP200 core and

577 the monolithic composite. Thus, low density cores lead to lower values of deflection as well as

578 lower transmitted impulse at all impulse intensities.
(a) Normalized Deflection $\quad \Delta / L$
(b) Transmitted Impulse $I_{B}(\mathrm{KPa} \cdot \mathrm{s})$
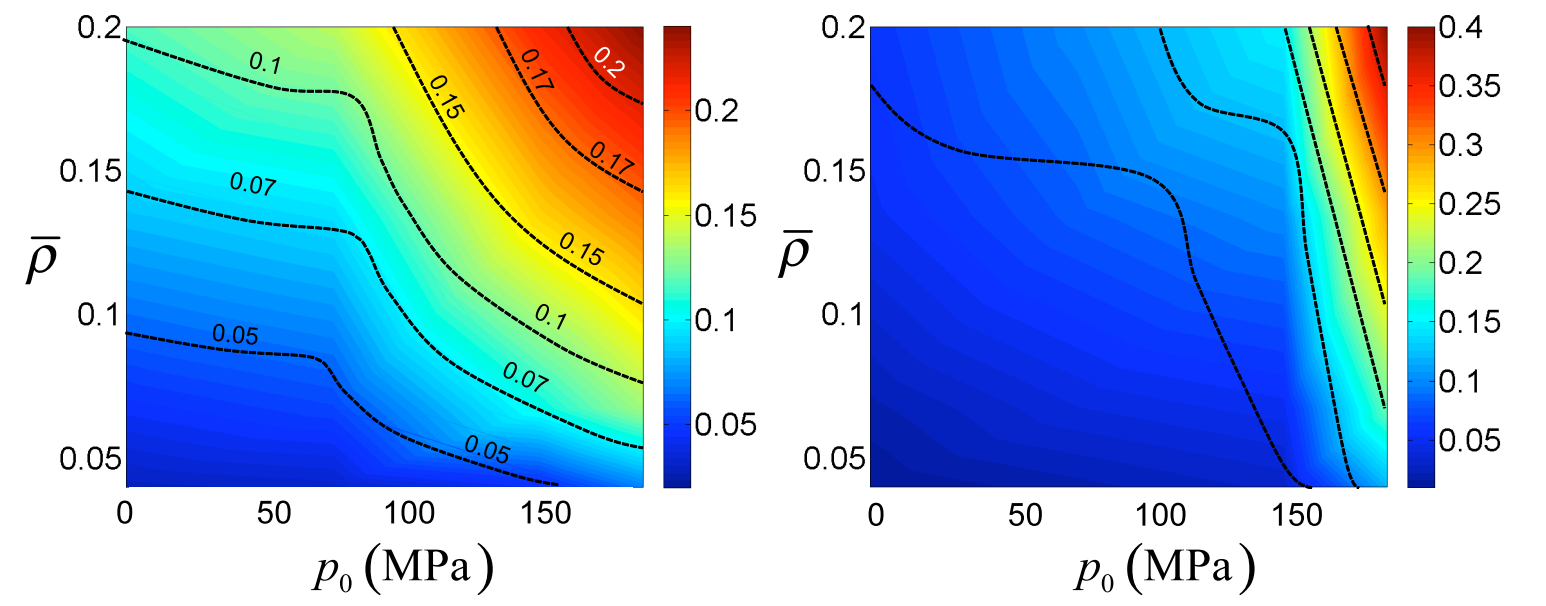

580 Figure 25 Loading-structure-performance maps for simply-supported sandwich plates showing 581 (a) deflection and (b) transmitted impulse as functions of peak pressure $p_{0}$ and normalized 582 density $\bar{\rho}$. 
584 Figure 15) are compared with those for the simply-supported bend loading configuration (Figure 585 25) to gain insight into the role of core density on bending and failure. Higher core densities $586(0.06<\bar{\rho}<0.1)$ limit core crushing, enable higher energy absorption and help maintain the 587 bending strength of a sandwich plate, but result in significantly more momentum being imparted 588 to the structure. This leads to more severe core damage and out-of-plane deflection. Conversely, 589 lower density cores $(0.01<\bar{\rho}<0.06)$ are susceptible to collapse under high intensity loads 590 which can have adverse effects on survivability and residual bending strength. The trade-off 591 between core compression and impulse transmission needs to be considered depending on 592 application. In marine structures supported by stiffeners with water on the impulse side and air 593 on the downstream side, the core has to provide load spreading and impulse absorption 594 capabilities. Since an air-backed sandwich structure is free to deform in the out-of-plane 595 direction, low density cores with $0.01<\bar{\rho}<0.06$ can satisfy both requirements $[13,37,38]$. Performance in water-backed conditions is important for the design of parts of ship 597 structures like turbine blades, hull and keel. Water-backed conditions also prevail in underwater 598 pipelines and ducts. In these cases, damage is localized and the structure is relatively undamaged 599 in regions that are away from the loading area. Tensile loads and fracture in both faces are 600 negligible due to the lack of overall deflection and bending, but the cores undergo severe 601 compression $[13,37,38]$. Consequently, higher core densities are essential in order to maintain 602 structural integrity in case of a blast event. Since minimizing core compression is essential in 603 water-backed conditions, core densities with $0.06<\bar{\rho}<0.1$ are most optimal because data in 604 Figure $13(\mathrm{a}-\mathrm{d})$ for $0.01<\bar{\rho}<0.06$ suggest that structures are susceptible to collapse under high 605 intensity loads. 


\section{Concluding remarks}

The conclusions of this study relating to the load-carrying and blast mitigation capacities of 608 sandwich plates with polymeric foam cores are as follows:

609 1. The compressive strain experienced by and the impulse transmitted by the sandwich core pose opposing requirements on structural design. Low density cores experience high compressive strains while transmitting lower impulses. On the other hand, high density cores behave like monolithic plates and transmit large fractions of the incident impulse. Although low density cores transmit significantly lower impulses, it should be noted that the kinetic energy acquired by frontfaces in low density cores is much higher, leading to severe core compression. This increased core compression is detrimental to bending stiffness and strength. Structural design must balance the competing requirements.

2. Experiments and simulations are in reasonable agreement in terms of the extent of core compression and impulse transmission. Over the range of impulses and structural configurations considered, the finite element predictions are within $10 \%$ of the experimental data. The homogenized, crushable foam constitutive model employed provides accurate tracking of the early stage response of the core material. However, the model slightly overestimates the compliance of the core leading to an increase in core compressive strain and a decrease in the transmitted impulses in comparison to experiments. The numerical calculations have provided an in-depth understanding of the temporal and spatial evolution of deformation modes in the core material.

3. Cores with different densities show significantly different deformation behaviors. Low density cores like HP60, HP100 and HP130 $(0.01<\bar{\rho}<0.06)$ experience rather uniform straining throughout their thickness and provide high impulse mitigation capacity. High 
density cores such as HP200 and HP250 $(0.06<\bar{\rho}<0.1)$ experience strain localizations that occur primarily near the facesheets. Such non-uniform distribution of straining leads to high impulse transfer and severe damage in the core material.

4. Loading-structure-performance maps derived from uniaxial compressive loading are compared to those obtained from the simply-supported bend loading configuration to offer insight into the role of core density on bending and failure of sandwich structures. The relative core density is found to be an important parameter determining the performance of sandwich structures in simply-supported conditions. Greater core compressibility minimizes both the deflection and impulse transmission in this configuration. Higher core densities $(0.06<\bar{\rho}<0.1)$ limit core crushing, enable higher energy absorption and help maintain the bending strength of a sandwich plate, but result in significantly more momentum being imparted to the structure. This leads to higher core damage and out-of-plane deflection. Conversely, lower density cores $(0.01<\bar{\rho}<0.06)$ are susceptible to collapse under high intensity loads which can have adverse effects on survivability and residual bending strength.

5. The frontface and backface masses are varied independently and results indicate that the frontface mass has a significant influence on core compression and impulse transmission, while the backface mass has a negligible effect on structural response. The momentum transmitted into the sandwich plates with $\Delta T_{f} / T_{c}<0.1$ is substantially lower than that for $\Delta T_{f} / T_{c}>0.15$. For the same core density, a $100 \%$ increase in facesheet thickness leads to a $25 \%$ and $50 \%$ increase in the core strain and normalized transmitted impulse, respectively. The greatest momentum transfer occurs in the case of monolithic plates of 
equivalent mass as sandwich plates. For a given incident impulse, $\Delta T_{f} / T_{c}>0.15$ results

in severe core compression and collapse because the impulse acquired by the frontface increases in proportion to mass.

In this combined experimental and numerical study, a parametric approach is employed

655 to develop loading-structure-performance maps to quantify core compression, deflection and

656 impulse transmission as a function of incident load (air-backed or water-backed conditions, load

657 intensity), structural attributes, and loading configurations. The insight gained here provides

658 guidelines for the design of structures for which response to water-based impulsive loading is an

659 important consideration.

\section{Acknowledgements}

Support by the Office of Naval Research through grant numbers N00014-09-1-0808 and

N00014-09-1-0618 (program manager: Dr. Yapa D. S. Rajapakse) is gratefully acknowledged.

663 Calculations are carried out on the Athena HPC cluster in the Dynamic Properties Research

664 Laboratory at Georgia Tech. MZ also acknowledges beneficial interactions through the

665 CAS/SAFEA International Partnership Program for Creative Research Teams.

666 10. References

667 [1] C.A. Steeves, N.A. Fleck, Collapse mechanisms of sandwich beams with composite faces 668 and a foam core, loaded in three-point bending. Part II: experimental investigation and numerical 669 modelling, International Journal of Mechanical Sciences, 46 (2004) 585-608.

670 [2] V.L. Tagarielli, V.S. Deshpande, N.A. Fleck, The dynamic response of composite sandwich 671 beams to transverse impact, International Journal of Solids and Structures, 44 (2007) 2442-2457.

672 [3] P.M. Schubel, J.J. Luo, I.M. Daniel, Impact and post impact behavior of composite sandwich 673 panels, Composites Part A-Applied Science And Manufacturing, 38 (2007) 1051-1057.

674 [4] J.A. Nemes, K.E. Simmonds, Low-Velocity Impact Response of Foam-Core Sandwich 675 Composites, Journal of Composite Materials, 26 (1992) 500-519.

676 [5] R.A.W. Mines, C.M. Worrall, A.G. Gibson, The Static and Impact Behavior of Polymer 677 Composite Sandwich Beams, Composites, 25 (1994) 95-110.

678 [6] I.M.D. J. L. Abot, Composite sandwich beams under low velocity impact, Proc. of AIAA 679 Conf., Seattle, (2001). 
[7] P.M. Schubel, J.J. Luo, I.M. Daniel, Low velocity impact behavior of composite sandwich panels, Composites Part a-Applied Science and Manufacturing, 36 (2005) 1389-1396.

682 [8] J.W. Hutchinson, Z.Y. Xue, Metal sandwich plates optimized for pressure impulses, International Journal of Mechanical Sciences, 47 (2005) 545-569.

684 [9] Y.M. Liang, A.V. Spuskanyuk, S.E. Flores, D.R. Hayhurst, J.W. Hutchinson, R.M. 685 McMeeking, A.G. Evans, The response of metallic sandwich panels to water blast, Journal Of 686 Applied Mechanics-Transactions Of The Asme, 74 (2007) 81-99. [10] Z.Y. Xue, J.W. Hutchinson, Preliminary assessment of sandwich plates subject to blast loads, International Journal of Mechanical Sciences, 45 (2003) 687-705. [11] Z.Y. Xue, J.W. Hutchinson, A comparative study of impulse-resistant metal sandwich plates, International Journal of Impact Engineering, 30 (2004) 1283-1305.

691 [12] S. Avachat, M. Zhou, Effect of Facesheet Thickness on Dynamic Response of Composite 692 Sandwich Plates to Underwater Impulsive Loading, Experimental Mechanics, Volume 52 (2011) 693 pp 83-93.

694 [13] S. Avachat, M. Zhou, Dynamic Response Of Composite Sandwich Structures Subjected To 695 Underwater Impulsive Loads: Experiments And Simulations Conference Proceedings of the 16th 696 International Conference on Composite Structures, ICCS-16, A. J. M. Ferreira (Editor), FEUP, 697 Porto, 2011, (2011).

698 [14] S. Avachat, M. Zhou, Dynamic Response of Submerged Composite Sandwich Structures to Blast Loading, Proceedings of the IMPLAST 2010 - SEM Fall Conference, October 12-14 2010 700 Providence, Rhode Island, USA, Arun Shukla (Editor), (2010).

701 [15] F. Latourte, D. Gregoire, D. Zenkert, X.D. Wei, H.D. Espinosa, Failure mechanisms in 702 composite panels subjected to underwater impulsive loads, Journal of the Mechanics and Physics

703 of Solids, 59 (2011) 1623-1646.

[16] X.D. Wei, P. Tran, A. de Vaucorbeil, R.B. Ramaswamy, F. Latourte, H.D. Espinosa, Threedimensional numerical modeling of composite panels subjected to underwater blast, Journal of the Mechanics and Physics of Solids, 61 (2013) 1319-1336.

[17] M.M. Swisdak, Explosion effects and properties: Part II - explosion effects in water, Technical Report, Naval Surface Weapons Center, Dahlgren, Virginia, USA, (1978).

[18] G.I. Taylor, The pressure and impulse of submarine explosion waves on plates. , The scientific papers of G I Taylor, vol. III, pp. 287-303. Cambridge: Cambridge University Press, (1941).

[19] H. Arora, M. Kelly, A. Worley, P. Del Linz, A. Fergusson, P.A. Hooper, J.P. Dear, Compressive strength after blast of sandwich composite materials, Philosophical Transactions of the Royal Society a-Mathematical Physical and Engineering Sciences, 372 (2014).

[20] G.I. Taylor, The Scientific Papers of G I Taylor, Cambridge University Press, Cambridge, (1963).

[21] N. Kambouchev, R. Radovitzky, L. Noels, Fluid-structure interaction effects in the dynamic response of free-standing plates to uniform shock loading, Journal of Applied MechanicsTransactions of the Asme, 74 (2007) 1042-1045.

[22] J.W. Hutchinson, Energy and Momentum Transfer in Air Shocks, Journal of Applied Mechanics-Transactions of the Asme, 76 (2009).

[23] R.H. Cole, Spherical Shock Waves from Underwater Explosions, Physical Review, 72 (1947) 177-177. 
[24] N. Kambouchev, L. Noels, R. Radovitzky, Numerical simulation of the fluid-structure interaction between air blast waves and free-standing plates, Computers \& Structures, 85 (2007) 923-931.

[25] T. George, V.S. Deshpande, K. Sharp, H.N.G. Wadley, Hybrid core carbon fiber composite sandwich panels: Fabrication and mechanical response, Composite Structures, 108 (2014) 696710. [26] S.D. DIAB Inc., DeSoto, Texas 75115, USA.

[27] Hibbit, Karlsson, Sorensen, Abaqus/Explicit User's Manual, Version 6.9, (2009).

[28] V.S. Deshpande, N.A. Fleck, Multi-axial yield behaviour of polymer foams, Acta Materialia, 49 (2001) 1859-1866.

[29] H. Hooputra, H. Gese, H. Dell, H. Werner, A comprehensive failure model for crashworthiness simulation of aluminium extrusions, Int J Crashworthines, 9 (2004) 449-463. [30] J. Zhang, N. Kikuchi, V. Li, A. Yee, G. Nusholtz, Constitutive modeling of polymeric foam material subjected to dynamic crash loading, International Journal of Impact Engineering, 21 (1998) 369-386.

[31] V.S. Deshpande, N.A. Fleck, Isotropic constitutive models for metallic foams, Journal of the Mechanics and Physics of Solids, 48 (2000) 1253-1283.

[32] P. Poapongsakorn, L.A. Carlsson, Fracture toughness of closed-cell PVC foam: Effects of loading configuration and cell size, Composite Structures, 102 (2013) 1-8.

[33] A. Needleman, V. Tvergaard, Mesh Effects in the Analysis of Dynamic Ductile CrackGrowth, Engineering Fracture Mechanics, 47 (1994) 75-91.

[34] A.S. Gullerud, X.S. Gao, R.H. Dodds, R. Haj-Ali, Simulation of ductile crack growth using computational cells: numerical aspects, Engineering Fracture Mechanics, 66 (2000) 65-92.

[35] S. Avachat, M. Zhou, High-speed digital imaging and computational modeling of dynamic failure in composite structures subjected to underwater impulsive loads, International Journal of Impact Engineering, 77 (2015) 147-165.
[36]
S.D.
DIAB
Inc.,
DeSoto,
Texas
75115 ,
USA

http://www.diabgroup.com/europe/literature/e_pdf_files/man_pdf/H_man.pdf Accessed 5 May 2011.

[37] S. Avachat, M. Zhou, Effect of core density on deformation and failure in sandwich composites subjected to underwater impulsive loads, The International Journal of Multiphysics Volume 6 (2012).

[38] S. Avachat, M. Zhou, Dynamic Response of Submerged Composite Sandwich Structures to Blast Loading, in: A. Shukla (Ed.) IMPLAST 2010 - SEM Fall Conference, Society for 759 